Pacific Journal of Mathematics

UNIQUENESS PROBLEM WITHOUT MULTIPLICITIES IN 


\title{
UNIQUENESS PROBLEM WITHOUT MULTIPLICITIES IN VALUE DISTRIBUTION THEORY
}

\author{
SHANYU JI
}

Let $H_{1}, \ldots, H_{k}$ be hyperplanes in general position in $\mathbf{P}^{m}$ with $m \geq$ 2. Let $A_{1}, \ldots, A_{k}$ be pure $(n-1)$-dimensional analytic subsets of $\mathbf{C}^{n}$ with $\operatorname{codim} A_{i} \cap A_{j} \geq 2$ whenever $i \neq j$. Then any linearly nondegenerate meromorphic maps $f, g, h: \mathbf{C}^{n} \rightarrow \mathbf{P}^{m}$ with $f\left|A_{j}=g\right| A_{j}=$ $h \mid A_{j}$ and with $f^{-1}\left(H_{j}\right)=g^{-1}\left(H_{\jmath}\right)=h^{-1}\left(H_{J}\right)=A_{J}$ for $j=1, \ldots, k$ satisfy Property (P) if $k=3 m+1$. Consequently such $f, g, h$ are algebraically dependent. If even $n \geq \operatorname{rank} f=\operatorname{rank} g=\operatorname{rank} h=m$, then $k=m+3$ suffices.

1. Introduction. Since Pólya's work [P1] in 1929, the uniqueness problem in value distribution theory has been studied by Nevanlinna [N1], Cartan [C1, C2], Fujimoto [F1, F2], Schmid [S1], Smiley [S5, S6], Carlson [D2], Drouilhet [D1, D2] and Stoll [S11]. One of the main results, given by Fujimoto in 1979 [F2], is that if $H_{j}$ are hyperplanes in $\mathbf{P}^{m}$ in general position and $v_{j}$ are divisors on $\mathbf{C}^{n}$ whose supports have no common irreducible components for $j=1, \ldots, m+2$ and if $\mathscr{W}$ is the set of meromorphic maps $f: \mathbf{C}^{n} \rightarrow \mathbf{P}^{m}$ with $f^{*}\left(H_{j}\right)=v_{j}$ for $j=1, \ldots, m+2,\left(f^{*}\left(H_{j}\right)\right.$ is the pull-back of the divisor of $H_{j}$ on $\mathbf{P}^{m}$ by $f$ ), then $\mathscr{W}$ cannot contain more than $m+1$ algebraically independent maps. This theorem is in fact a generalization of the Cartan-Nevanlinna theorem (i.e., take $n=m=1$ and replace "algebraically dependent maps" by "maps" in the above theorem) in 1928 .

In this paper, we shall give some analogous results which are without multiplicities. For this kind of problem, Cartan declared [C2] that there are at most two meromorphic functions $f, g$ on $\mathbf{C}$ such that $f^{-1}\left(a_{j}\right)=g^{-1}\left(a_{j}\right)$ for four distinct $a_{j} \in \mathbf{P}^{1}$. Cartan's proof appears to have a gap. But some of his original ideas are used in this paper. We also need to use some of Shiffman's and Drouilhet's results [S3], [D2].

Let $H_{1}, \ldots, H_{k}$ be hyperplanes in general position in $\mathbf{P}^{m}$ given by

$$
a_{0}^{(j)} w_{0}+\cdots+a_{m}^{(j)} w_{m}=0
$$

for $j=1, \ldots, k$. Let $A_{1}, \ldots, A_{k}$ be pure $(n-1)$-dimensional analytic subsets of $\mathbf{C}^{n}$ with $\operatorname{codim} A_{i} \cap A_{j} \geq 2$ whenever $i \neq j$. Put 
$A:=A_{1} \cup \cdots \cup A_{k}$. Let $F: \mathbf{C}^{n} \rightarrow \mathbf{P}^{m}$ be a meromorphic map. Let

(1.2) $\mathscr{F}_{B}\left(\mathbf{C}^{n}, \mathbf{P}^{m}, k\right)$

$$
\begin{aligned}
:=\left\{f: \mathbf{C}^{n} \rightarrow \mathbf{P}^{m} \mid f\right. & \text { is a linearly non-degenerate } \\
& \text { meromorphic map, } f|A=F| A \\
& \text { and } \left.f^{-1}\left(H_{j}\right)=A_{j} \text { for } j=1, \ldots, k\right\}
\end{aligned}
$$

(for the definition of linearly non-degenerate, see $\S 2$ ) and

$$
\mathscr{F}_{A}\left(\mathbf{C}^{n}, \mathbf{P}^{m}, k\right):=\left\{f \in \mathscr{F}_{B}\left(\mathbf{C}^{n}, \mathbf{P}^{m}, k\right) \mid \operatorname{rank} f=m\right\} .
$$

We say that $f, g, h \in \mathscr{F}_{B}\left(\mathbf{C}^{n}, \mathbf{P}^{m}, k\right)$ satisfy Property (P) if for each fixed $p=\left(p_{0}, \ldots, p_{m}\right) \in \mathbf{C}_{*}^{m+1}:=\mathbf{C}^{m+1}-\{0\}$, for all but at most four values of $i$ in the set $\{1, \ldots, k\}$, there exist $\left(\lambda_{i}, \mu_{i}, \nu_{i}\right) \in \mathbf{C}_{*}^{3}=\mathbf{C}^{3}-\{0\}$, such that

$$
\begin{aligned}
\frac{\lambda_{i}\left(p_{0} f_{0}+\cdots+p_{m} f_{m}\right)}{a_{0}^{(i)} f_{0}+\cdots+a_{m}^{(i)} f_{m}} & +\frac{\mu_{i}\left(p_{0} g_{0}+\cdots+p_{m} g_{m}\right)}{a_{0}^{(i)} g_{0}+\cdots+a_{m}^{(i)} g_{m}} \\
& +\frac{\nu_{i}\left(p_{0} h_{0}+\cdots+p_{m} h_{m}\right)}{a_{0}^{(i)} h_{0}+\cdots+a_{m}^{(i)} h_{m}}=0
\end{aligned}
$$

where $\left(f_{0}, \ldots, f_{m}\right),\left(g_{0}, \ldots, g_{m}\right)$, and $\left(h_{0}, \ldots, h_{m}\right)$ are reduced representations of $f, g, h$, respectively, and $a_{0}^{(i)}, \ldots, a_{m}^{(i)}$ are given by $(1.1)$. (The set of omitted values of $i$ depends on $p$.)

Our main results are

Theorem A. If $f, g, h \in \mathscr{F}_{A}\left(\mathbf{C}^{n}, \mathbf{P}^{m}, m+3\right)$ with $m \geq 2$, then $f, g, h$ satisfy Property (P). Consequently $f, g, h$ are algebraically dependent.

THEOREM B. If $f, g, h \in \mathscr{F}_{B}\left(\mathbf{C}^{n}, \mathbf{P}^{m}, 3 m+1\right)$ with $m \geq 2$, then $f, g, h$ satisfy Property $(\mathrm{P})$. Consequently $f, g, h$ are algebraically dependent.

Remark. By [D1], if $k>m+3, \mathscr{F}_{A}\left(\mathbf{C}^{n}, \mathbf{P}^{m}, k\right)$ contains at most one map. By [S5, S6], if $k>3 m+1, \mathscr{F}_{B}\left(\mathbf{C}^{n}, \mathbf{P}^{m}, k\right)$ contains at most one map.

As generalization of Theorem $\mathrm{A}$, we have Theorem $\mathrm{C}$ as follows.

Let $V \subset \mathbf{P}^{M}$ be a connected complex submanifold with $\operatorname{dim} V=$ $m \geq 2$. Assume that $V$ is not contained in any hyperplane of $\mathbf{P}^{M}$. Let $H_{1}, \ldots, H_{k}$ be hyperplanes of $\mathbf{P}^{M}$ such that $D_{j}:=H_{j} \cap V$ are pure $(m-1)$-dimensional smooth analytic subsets of $V$. Let $D_{1}, \ldots, D_{k}$ have normal crossings in $V$. Let $A_{1}, \ldots, A_{k}$ be pure $(n-1)$-dimensional analytic subsets of $\mathbf{C}^{n}$ with $\operatorname{codim} A_{i} \cap A_{j} \geq 2$ whenever $i \neq j$. Put 
$A:=A_{1} \cup \cdots \cup A_{k}$. Suppose $k$ is an integer such that $K_{V} \otimes(H \mid V)^{k-2} \geq 0$ and $k \geq 5$, where $K_{V}$ is the canonical bundle of $V$ and $H$ is the hyperplane section bundle on $\mathbf{P}^{M}$. Let $F: \mathbf{C}^{n} \rightarrow V$ be a meromorphic map. Let

$$
\begin{aligned}
& \mathscr{F}_{C}\left(\mathbf{C}^{n}, V, k\right) \\
& \quad:=\left\{f: \mathbf{C}^{n} \rightarrow V \text { is meromorphic } \operatorname{rank} f=m,\right. \\
& \left.\quad f|A=F| A \text { and } f^{-1}\left(D_{j}\right)=A_{j} \text { for } j=1, \ldots, k\right\} .
\end{aligned}
$$

Since $f: \mathbf{C}^{n} \rightarrow V$ can be regarded as $f: \mathbf{C}^{n} \rightarrow \mathbf{P}^{M}$ by composing with the inclusion map: $V \hookrightarrow \mathbf{P}^{M}$. So, $\mathscr{F}_{C}\left(\mathbf{C}^{n}, V, k\right)=\mathscr{F}_{C}\left(\mathbf{C}^{n}, V, k\right) \cap$ $\mathscr{F}_{B}\left(\mathbf{C}^{n}, \mathbf{P}^{M}, k\right)$. We say $f, g, h \in \mathscr{F}_{C}\left(\mathbf{C}^{n}, V, k\right)$ satisfy Property $(\mathbf{P})$ if $f$, $g, h$ as maps in $\mathscr{F}_{B}\left(\mathbf{C}^{n}, P^{M}, k\right)$ satisfy Property $(\mathbf{P})$.

Theorem C. If $f, g, h \in \mathscr{F}_{C}\left(\mathbf{C}^{n}, V, k\right)$, then $f, g, h$ satisfy Property (P). Consequently $f, g, h$ are algebraically dependent.

REMARK. (i) When $V=\mathbf{P}^{M}$, Theorem $\mathrm{C}$ is Theorem A.

(ii) By [D2], if $K_{V} \otimes(H \mid V)^{K-2}>0$, then $\mathscr{F}_{C}\left(\mathbf{C}^{n}, V, k\right)$ contains at most one map.

The author would like to thank his advisor, B. Shiffman, for encouragement and assistance. The author also would like to thank Professor W. Stoll for giving very careful and helpful corrections and suggestions for my manuscript.

2. Preliminaries. We give or review some notions and definitions (cf. $[\mathbf{S 7}, \mathbf{S 8}]$ ).

DEFINITION 2.1. (For meromorphic maps.)

(a) Let $X$ and $Y$ be connected complex manifolds. Let $S$ be a proper analytic subset of $X$. Then $X-S$ is dense open in $X$. Let $f: X-S \rightarrow Y$ be a holomorphic map. The closed graph of $f$ is the closure $\Gamma(f):=\overline{\{(x, f(x)) \mid x \in X-S\}}$ of the graph of $f$ over $X-$ $S$ in $X \times Y$. Let $\pi: \Gamma(f) \rightarrow X$ and $\hat{f}: \Gamma(f) \rightarrow Y$ be the natural projections. The map $f$ is said to be meromorphic on $X$ if $\Gamma(f)$ is analytic in $X \times Y$ and $\pi$ is proper. Let $\# P$ be the cardinality of $P$. The indeterminacy $I_{f}:=\left\{x \in X \mid \# \hat{f}\left(\pi^{-1}(x)\right)>1\right\}$ is analytic and contained in $S$. Codim $I_{f} \geq 2$. We can assume $S=I_{f}$. In this paper, we always assume that $Y$ is algebraic and compact.

(b) Let $V$ be a complex vector space of dimension $m+1$. Put $V_{*}=V-\{0\}$. Then $\mathbf{C}_{*}=\mathbf{C}-\{0\}$ acts by multiplication on $V_{*}$. The quotient space $\mathbf{P}(V):=V_{*} / \mathbf{C}_{*}$ is the projective space associated 
to $V$. Let $\mathbf{P}: V_{*} \rightarrow P(V)$ be the residual map. If $A \subseteq V$, define $\mathbf{P}(A):=\{\mathbf{P}(a) \mid 0 \neq a \in A\}$. Let $S$ and $X$ be as in (a). Let $f: X-$ $S \rightarrow \mathbf{P}(V)$ be a holomorphic map. Let $x \in X$ and $U$ be an open connected neighborhood of $x$. A holomorphic map $\phi_{f}: U \rightarrow V$ is called a representation of $f$ at $x$ if $\phi_{f} \not \equiv 0$ and if $f(z)=\mathbf{P}\left(\phi_{f}(z)\right)$ for all $z \in U-S$ with $\phi_{f}(z) \neq 0$. The representation is said to be reduced if $\operatorname{Codim} \phi_{f}^{-1}(0) \geq 2$. The map $f$ is meromorphic if and only if there is a representation at every point of $X$. The representation is global if $U=X$. If $X=\mathbf{C}^{n}$, there is a global reduced representation. So, maps in $\mathscr{F}_{B}\left(\mathbf{C}^{n}, \mathbf{P}^{m}, k\right)$ have global representations.

(c) We have assumed that $Y$ is an algebraic compact manifold. A meromorphic map $f: X \rightarrow Y$ is called algebraically non-degenerate if the image of $f$ is not contained in any proper analytic subset of $Y$. If $Y=\mathbf{P}^{m}$, then $f$ is called linearly non-degenerate if the image of $f$ is not contained in any hyperplane of $\mathbf{P}^{m}$.

(d) Meromorphic maps $f^{1}, \ldots, f^{k}$ of $\mathbf{C}^{n}$ into any algebraic manifold $Y$ are called algebraically dependent if the image of the meromorphic map $f^{1} \times \cdots \times f^{k}: \mathbf{C}^{n} \rightarrow Y \times \cdots \times Y$ ( $k$ times) is contained in some proper analytic subset of $Y \times \cdots \times Y$ ( $k$ times), where $\left(f^{1} \times \cdots \times f^{k}\right)(z):=\left(f^{1}(z), \ldots, f^{k}(z)\right)$ for generic points $z$ in $\mathbf{C}^{n}$.

(e) Let $X$ be as in (a). Let $\mathscr{O}$ be the sheaf of germs of holomorphic functions on $X$. If $x \in X$, let $\mathscr{O}_{x}$ be the stalk of $\mathscr{O}$ over $x$, and $m_{x}$ be the maximal ideal in $\mathscr{O}_{x}$. Let $m_{x}^{p}$ be its $p$ th power with $m_{x}^{0}:=\mathscr{O}_{x}$. Take $0 \neq f \in \mathscr{O}_{x}$, one and only one integer $\mu(f) \geq 0$ exists such that $f \in m_{x}^{\mu(f)}-m_{x}^{\mu(f)+1}$. Here $\mu(f)$ is called the zero-multiplicity of $f$. Let $U \subset X$ be open connected, $f \neq \equiv 0$ be holomorphic function on $U$. Take any $x \in U$ and let $f_{x} \in \mathscr{O}_{x}$ be the germ defined by $f$ in $\mathscr{O}_{x}$. Then $\mu_{f}^{0}(x):=\mu\left(f_{x}\right) \geq 0$ is called the zero-multiplicity of $f$ at $x$. The function $\mu_{f}^{0}: U \rightarrow \mathbf{Z}$ is called the zero divisor of $f$.

A divisor $v$ on $X$ is an integral value function $v: X \rightarrow \mathbf{Z}$ such that for every $x \in X$ there exists an open connected neighborhood $U$ of $x$ and holomorphic functions $g \not \equiv 0 \not \equiv$ on $U$ with

$$
\operatorname{Codim}\left(g^{-1}(0) \cap h^{-1}(0)\right) \geq 2 \text {, }
$$

such that $v \mid U=\mu_{g}^{0}-\mu_{h}^{0} . v \equiv 0$ is called the null divisor. If $v$ is not the null divisor, $S:=\operatorname{supp} v$ is a pure $(n-1)$-dimensional analytic subset of $X$ where $\operatorname{dim} X=n$. Let $\operatorname{Reg}(S)$ be the set of regular points of $S$. Then $v \mid \operatorname{Reg}(S)$ is locally constant. Let $L$ be the set of irreducible components of $S$. For each $B \in L, v \mid \operatorname{Reg}(S) \cap B \equiv p(v, B) \not \equiv 0$ is a constant integer. We say that $v \geq 0$ if $p(v, B) \geq 0$ for all $B \in L$. 
For any pure $(n-1)$-dimensional analytic subset $A$ of $X$, there exists a unique divisor $v_{A}$ such that $v_{A} \mid \operatorname{Reg}(A) \equiv 1$. Obviously $v_{A} \geq 0$. The locally finite sum

$$
v=\sum_{B \in L} p(v, B) v_{B}
$$

is called the analytic chain representation of $v$. If $0 \leq n \in \mathbf{Z}$, the divisor

$$
v^{(n)}:=\sum_{B \in L} \min (n, p(v, B)) v_{B}
$$

is called the truncation of $v$ at level $n$.

Let $f: X \rightarrow Y$ be meromorphic. Let $v: Y \rightarrow Z$ be a divisor on $Y$ with $S=\operatorname{supp} v$. Assume that $f(X) \nsubseteq S$. Since Codim $I_{f} \geq 2$, there exists a unique divisor $f^{*}(v)$ on $X$, called the pull-back divisor of $v$ by $f$, such that for any pair of open, connected, non-empty subsets $U$ of $X-I_{f}$ and $W$ of $Y$ with $f(U) \subseteq W$, there are holomorphic functions $g \not \equiv 0 \not \equiv h$ on $W$ with $\operatorname{Codim}\left(g^{-1}(0) \cap h^{-1}(0)\right) \geq 2$ and $v \mid W=\mu_{g}^{0}-\mu_{h}^{0}$, then $g \circ f|U \not \equiv 0 \not \equiv h \circ f| U$, and $f^{*}(v)\left|U=\left(\mu_{g \circ f}^{0}-\mu_{h \circ f}^{0}\right)\right| U$.

DEFINITION 2.2. (For Nevanlinna theory.)

(a) For $z=\left(z_{1}, \ldots, z_{n}\right) \in \mathbf{C}^{n}$, let $\left\{\left.z\right|^{2}:=\left|z_{1}\right|^{2}+\cdots+\left|z_{n}\right|^{2} . S(r):=\right.$ $\left\{z \in \mathbf{C}^{n}|| z \mid=r\right\} . B(r):=\left\{z \in \mathbf{C}^{n}|| z \mid<r\right\} . d^{c}:=(\partial-\bar{\partial}) / 4 \pi \sqrt{-1}$. Let $\omega_{0}:=d d^{c} \log |z|^{2}$ be the homogeneous metric form on $\mathbf{C}^{n}$, and $\varphi_{0}:=d d^{c}|z|^{2}$ be the Euclidean metric form on $\mathbf{C}^{n}$. Let $\sigma:=d^{c} \log |z| \wedge$ $\omega_{0}^{n-1}$ be the Poincare form on $S(r)$. Let $\omega$ be the Fubini-Study metric form on $\mathbf{P}^{n-1}$ given by $\mathbf{P}^{*} \omega=\omega_{0}$. Denote $\mathbf{C}_{*}^{n}=\mathbf{C}^{n}-\{0\}$.

(b) Let $v$ be a divisor on $\mathbf{C}^{n}$ with $S=\operatorname{supp} v$. For $t>0$, the counting function $n_{v}$ is defined by

$$
n_{v}(t):= \begin{cases}\sum_{z \in S \cap B(t)} v(z), & \text { if } n=1, \\ \frac{1}{t^{2 n-1}} \int_{S \cap B(t)} v \varphi_{0}^{n-1}, & \text { if } n>1 .\end{cases}
$$

If $n>1$, also $n_{v}(t)=\int_{S \cap B(t)} v \omega_{0}^{n-1}+v(0)$. For $0<s<r$, the integrate counting function is defined by

$$
N(v ; r, s):=\int_{s}^{r} n_{v}(t) \frac{d t}{t}
$$

If $f: \mathbf{C}^{n} \rightarrow Y$ is a meromorphic map, and $v$ is a divisor on $Y$ with $f\left(\mathbf{C}^{n}\right) \nsubseteq \operatorname{supp} v$, and $f^{*} v$ is the pull-back divisor of $v$ by $f$, we abbreviate $N\left(f^{*} v ; r, s\right)=N_{f}(v ; r, s)$. If $A$ is a pure 1-codimensional analytic 
subset of $Y$, abbreviate $N\left(f^{*}\left(v_{A}\right) ; r, s\right)=N_{f}(A ; r, s)$ and $N\left(v_{A} ; r, s\right)=$ $N(A ; r, s)$.

(c) Let $f: \mathbf{C}^{n} \rightarrow Y$ be meromorphic. Let $L$ be a hermitian holomorphic line bundle over $Y$ with a metric $h$. The characteristic function of $f$ is defined by

$$
A_{f}(L ; t)=A_{f}(L, h ; t):=\frac{1}{t^{2 n-2}} \int_{B(t)} f^{*}\left(c_{h}\right) \wedge \varphi_{0}^{n-1}, \text { for } t>0,
$$

(2.5) $\quad T_{f}(L ; r, s)=T_{f}(L, h ; r, s):=\int_{s}^{r} A_{f}(L ; t) \frac{d t}{t}, \quad$ for $0<s<r$

Let $A_{f}(L ; 0)=\lim _{t \rightarrow 0} A_{f}(L ; t)$. We also have

$$
A_{f}(L ; t)=\int_{B(t)} f^{*}\left(c_{h}\right) \wedge \omega_{0}^{n-1}+A_{f}(L ; 0), \quad \text { for } t \geq 0,
$$

where $c_{h}$ is the Chern form of the metric $h$ (Note: $A_{f}(L ; 0)=0$ if $f$ is holomorphic at 0 ).

If $f: \mathbf{C}^{n} \rightarrow \mathbf{P}^{m}$ is meromorphic, and $L$ is the hyperplane section bundle $H$ over $\mathbf{P}^{m}$ with the metric $h:=\left\{h_{\alpha}:=\left|z_{\alpha}\right|^{2} /\left(\left|z_{0}\right|^{2}+\cdots+\left|z_{m}\right|^{2}\right)\right.$ on $\left.U_{\alpha}\right\}$, where $U_{\alpha}:=\left\{\left[Z_{0}: \cdots: Z_{m}\right] \mid Z_{\alpha} \neq 0\right\}$ for $\alpha=0, \ldots, m$, then $c_{h}=\omega$. We abbreviate $T_{f}(H, h ; r, s)=T_{f}(r, s)$.

(d) Let $f: \mathrm{C}^{n} \rightarrow Y, L, h$ be as in (c), $Y$ compact. The set of all holomorphic sections in $L$ over $Y$ is a finite dimensional complex vector space $\Gamma(Y, L)$. Since $Y$ is compact, we can select a hermitian metric $l$ on $\Gamma(Y, L)$ such that $\|s(y)\|_{h} \leq\|s\|_{l}$ for all $s \in \Gamma(Y, L)$ and all $y \in Y$. If $y \in Y$, and $a \in \mathbf{P}(\Gamma(Y, L))$, then $a=\mathbf{P}(\tilde{a})$ with $0 \neq \tilde{a} \in$ $\Gamma(Y, L)$ and

$$
0 \leq\|y, a\|_{h, l}:=\frac{\|\tilde{a}(y)\|_{h}}{\|\tilde{a}\|_{l}} \leq 1
$$

is a well-defined function of $y$ and $a$. Let $a \in \mathbf{P}(\Gamma(Y, L))$ and assume $f\left(\mathbf{C}^{n}\right) \nsubseteq \operatorname{supp} \mu_{a}$, where $\mu_{a}$ is the associated divisor of $a$. Then $\|f, a\|_{h, l} \not \equiv 0$ and the proximity function

(2.7) $\quad m_{f}(a ; r)=m_{f}(a, L, h, l ; r):=\int_{S(r)} \log \frac{1}{\|f, a\|_{h, l}} \sigma>0$ exists.

If $Y=\mathbf{P}^{m}$, and $D$ is a hyperplane on $\mathbf{P}^{m}$ defined by $a_{0} w_{0}+\cdots+$ $a_{m} w_{m}=0$, assume $f\left(\mathbf{C}^{n}\right) \nsubseteq D$, since $D$ associates a unique element 
in $\mathbf{P}\left(\Gamma\left(\mathbf{P}^{m}, H\right)\right)$, where $H$ is as in (c),

(2.8) $m_{f}(D ; r)$

$$
\begin{aligned}
& :=-\int_{S(r)} \log \frac{\left|a_{0} f_{0}+\cdots+a_{m} f_{m}\right|}{\left(\left|f_{0}\right|^{2}+\cdots+\left|f_{m}\right|^{2}\right)^{1 / 2}\left(\left|a_{0}\right|^{2}+\cdots+\left|a_{m}\right|^{2}\right)^{1 / 2}} \sigma \\
& \geq 0
\end{aligned}
$$

where $\phi_{f}=\left(f_{0}, \ldots, f_{m}\right)$ is a reduced representation of $f$.

(e) The notation $\| A(r) \leq B(r)$ means that there is some set $E \subset$ $\mathbf{R}^{+}$with $\int_{E} d r<\infty$, such that $A(r) \leq B(r)$ for all $r \in \mathbf{R}^{+}-E$; $\| \lim _{r \rightarrow \infty} A(r)=B$ means that there is some subset $E \subset R^{+}$with $\int_{E} d r<\infty$, such that $\lim _{r \rightarrow \infty, r \in \mathbf{R}^{+}-E} A(r)=B ; \| A(r) \leq o(\log r)+B(r)$ means that there is some subset $E \subset \mathbf{R}^{+}$with $\int_{E} d r<\infty$, and a function $0 \leq \varepsilon: \mathbf{R}^{+} \rightarrow \mathbf{R}^{+}$with $\varepsilon(r) \rightarrow 0$ as $r \rightarrow+\infty$, such that $A(r) \leq \varepsilon(r) \log r+B(r)$ for all $r \in \mathbf{R}^{+}-E$.

(f) Sometimes we use $C$ to mean a positive constant number which is independent of $r \in \mathbf{R}^{+}$.

3. Lemma of the logarithmic derivative. In 1925, Nevanlinna proved the "lemma of the logarithmic derivative" [N2], [H1] in the form

$$
\| m_{f^{\prime} / f}(r) \leq 10 \log ^{+} T_{f}(r)+10 \log r+4 \log ^{+} \log ^{+}(1 /|f(0)|)
$$

for meromorphic function $f$ on $\mathbf{C}$. The generalization for it on $\mathbf{C}^{n}$ was given by Vitter in 1977 [V1]. Shiffman [S4] gave a refined lemma in which $O(\log r)$ is replaced by $o(\log r)$ :

LEMMA 3.1 ([S4, Lemma 3.11]). Let $F$ be a non-constant meromorphic function on $\mathbf{C}^{n}$. Then for any $i, 1 \leq i \leq n$ and $0<s<r$

$$
\| \int_{S(r)} \log ^{+}\left|\frac{\partial F}{\partial z_{i}} / F\right| \sigma \leq C \log ^{+}\left(T_{f}(r, s)\right)+o(\log r) .
$$

We need the following Lemma 3.2. The simplified proof of this lemma given below is due to W. Stoll.

For Lemma 3.2, some preparation is needed.

$\tilde{U}:=\left\{\left(\tilde{z}_{1}, \ldots, \tilde{z}_{n}\right) \in \mathbf{C}^{n} \mid \tilde{z}_{1} \neq 0\right\}$ is open in $\mathbf{C}^{n}$. Since $\tilde{U}=\mathbf{P}^{-1}(\mathbf{P}(\tilde{U}))$, the image $U:=\mathbf{P}(\tilde{U})$ is open in $\mathbf{P}^{n-1}$ and $\mathbf{P}^{n-1}-U=\mathbf{P}\left(\{0\} \times \mathbf{C}^{n-1}\right)$ is a hyperplane in $\mathbf{P}^{n-1}$. Now $E:=\{1\} \times \mathbf{C}^{n-1}$ is an affine plane in $\mathbf{C}^{n}$ of dimension $n-1$ with $E \subset \tilde{U}$. The restriction $\hat{\mathbf{P}}:=\mathbf{P}: E \rightarrow U$ is biholomorphic. If $z=\mathbf{P}(\tilde{z}) \in U$, then $\tilde{z}=\left(\tilde{z}_{1}, \ldots, \tilde{z}_{n}\right) \in \tilde{U}$ and $\hat{\mathbf{P}}^{-1}(z)=\tilde{z} / \tilde{z}_{1}$. Let

$$
L: E \rightarrow \mathbf{C}^{n}
$$


be the inclusion map. Let $\omega$ be the Fubini-Study form on $\mathbf{P}^{n-1}$. Then $L^{*}\left(\omega_{0}\right)=\hat{\mathbf{P}}^{*}(\omega)$. Define

$$
\tilde{u}_{j}:=(\underbrace{0, \ldots, 0}_{j-1}, 1,0, \ldots, 0) \in \mathbf{C}^{n} .
$$

Then $\tilde{u}_{1}, \ldots, \tilde{u}_{n}$ is an orthonormal base of $\mathbf{C}^{n}$. The dual base $\tilde{u}_{1}^{*}, \ldots, \tilde{u}_{n}^{*}$ of $\left(\mathbf{C}^{n}\right)^{*}$ is orthonormal. Put $u_{j}^{*}:=\mathbf{P}\left(\tilde{u}_{j}^{*}\right)$. Pick $x \in U$. Then $e=\left(e_{1}, \ldots, e_{n}\right)=\hat{\mathbf{P}}^{-1}(x) \times E$ satisfies $\tilde{u}_{1}^{*}(e)=e_{1}=1$ and $|e| \geq 1$. Hence

$$
\left\|x, u_{1}^{*}\right\|=\frac{\left|\tilde{u}_{1}^{*}(e)\right|}{|e|\left|\tilde{u}_{1}^{*}\right|}=\frac{1}{|e|} .
$$

Let $F: \mathbf{C}^{n} \rightarrow \mathbf{P}^{1}$ be a non-constant meromorphic function. Then $S:=\operatorname{supp} \mu_{F}^{0} \cup \operatorname{supp} \mu_{F}^{\infty}$ is empty or an analytic subset of pure dimension $n-1$ in $\mathbf{C}^{n}$, where $\mu_{F}^{\infty}:=\mu_{F^{-1}}^{0}, \phi_{F^{-1}}=\left(f_{1}, f_{0}\right)$ if $\phi_{F}=\left(f_{0}, f_{1}\right)$ is a reduced representation of $F$. Then $E_{0}:=\{e \in E \mid \mathrm{C} e \subseteq S\}$ has measure zero in $E$. Take any $e \in E-E_{0}$, a meromorphic function $F_{e} \not \equiv 0$ is defined by $F_{e}(w):=F(w e)$ for all $w \in \mathbf{C}$. Obviously, if $e=\left(e_{1}, \ldots, e_{n}\right) \in E-E_{0}$ then $e_{1}=1$ and

$$
F_{e}^{\prime}(w)=\sum_{i=1}^{n} e_{i} \frac{\partial F}{\partial z_{i}}(w e), \quad \text { for all } w \in \mathbf{C} .
$$

The function $g_{e}:=F_{e}^{\prime} / F_{e}$ is meromorphic on $\mathbf{C}$ for each $e \in E-E_{0}$.

LEMMA 3.2. For each $0<s<r \in \mathbf{R}^{+}$, the integral

$$
\int_{e \in E-E_{0}} m_{g_{e}}\left(\infty ; \frac{r}{|e|}\right) L^{*}\left(\omega_{0}^{n-1}\right)(e)
$$

exists with

$$
\begin{gathered}
\int_{e \in E-E_{0}} m_{g_{e}}\left(\infty ; \frac{r}{|e|}\right) L^{*}\left(\omega_{0}^{n-1}\right)(e) \\
\leq C\left(\log ^{+} T_{F}(r, s)\right)+o(\log r) .
\end{gathered}
$$

Proof. Take $e \in E-E_{0}$ and $w \in \mathbf{C}$ with $0 \neq F_{e}(w) \neq \infty$. Then $e=\left(e_{1}, \ldots, e_{n}\right)$ and

$$
\left|F_{e}^{\prime}(w)\right|^{2}=\left|\sum_{i=1}^{n} e_{i} \frac{\partial F}{\partial z_{i}}(w e)\right|^{2} \leq|e|^{2} \sum_{i=1}^{n}\left|\frac{\partial F}{\partial z_{i}}(w e)\right|^{2}
$$


or

$$
\begin{aligned}
\log ^{+}\left|g_{e}(w)\right| & =\log ^{+}\left|\frac{F_{e}^{\prime}(w)}{F_{e}(w)}\right| \\
& \leq \log |e|+\frac{1}{2} \log ^{+} \sum_{i=1}^{n}\left|\frac{\partial F}{\partial z_{i}}(w e)\right|^{2}|F(w e)|^{-2} \\
& \leq \log |e|+\sum_{i=1}^{n} \log ^{+}\left|\frac{\partial F}{\partial z_{i}}(w e) / F(w e)\right|+\frac{1}{2} \log n
\end{aligned}
$$

which implies

$$
\begin{aligned}
m_{g_{e}}\left(\infty ; \frac{r}{|e|}\right) & \\
\leq & \log |e|+\sum_{i=1}^{n} \frac{1}{2 \pi} \\
& \times \int_{0}^{2 \pi} \log ^{+}\left|\frac{\partial F}{\partial z_{i}}\left(r \exp (\sqrt{-1} \theta) \frac{e}{|e|}\right) / F\left(r \exp (\sqrt{-1} \theta) \frac{e}{|e|}\right)\right| d \theta \\
& +\frac{1}{2} \log n
\end{aligned}
$$

By $[\mathbf{W 1}$, p. 130, (2.12)] we have

$$
\begin{aligned}
\int_{e \in E-E_{0}} \log |e| L^{*}\left(\omega_{0}^{n-1}\right)(e) \\
=\int_{e \in E-E_{0}} \log \frac{1}{\left\|P(e), u_{1}^{*}\right\|} \hat{\mathbf{P}}^{*}\left(\omega^{n-1}\right)(e) \\
=\int_{x \in \mathbf{P}^{n-1}} \log \frac{1}{\left\|x, u_{1}^{*}\right\|} \omega^{n-1}(x)=\frac{1}{2} \sum_{\lambda=1}^{n-1} \frac{1}{\lambda} .
\end{aligned}
$$

A diffeomorphism $\Phi: E \times \mathbf{R}(0,2 \pi) \rightarrow \tilde{E}$ onto a subset $\tilde{E}$ of $S(r)$ is defined by $\Phi(e, \theta)=r \exp (\sqrt{-1} \theta) e /|e|$ such that $\tilde{E}$ is open in the topology of $S(r)$ and such that $S(r)-\tilde{E}$ has measure zero. Then

$$
\begin{aligned}
\int_{e \in E-E_{0}} & \frac{1}{2 \pi} \int_{0}^{2 \pi} \log ^{+}\left|\frac{\partial F}{\partial z_{i}}(\Phi(e, \theta)) / F(\Phi(e, \theta))\right| d \theta \hat{\mathbf{P}}^{*}(\omega)^{n-1}(e) \\
= & \int_{S(r)} \log ^{+}\left|\frac{\partial F}{\partial Z_{i}} / F\right| \sigma .
\end{aligned}
$$


Finally,

$$
\begin{aligned}
& \int_{e \in E-E_{0}} m_{g_{e}}\left(\infty ; \frac{r}{|e|}\right) L^{*}\left(\omega_{0}^{n-1}\right)(e) \\
& \leq \sum_{i=1}^{n} \int_{S(r)} \log ^{+}\left|\frac{\partial F}{\partial Z_{i}} / F\right| \sigma+\frac{1}{2} \log n+\sum_{\lambda=1}^{n-1} \frac{1}{\lambda}
\end{aligned}
$$

then (3.6) is proved by applying Lemma 3.1.

\section{Lemmas from Nevanlinna Main Theorems.}

LeMma 4.1. Let $f \in \mathscr{F}_{C}\left(\mathbf{C}^{n}, V, k\right), K_{V} \otimes(H \mid V)^{k-2} \geq 0$. Then for $0<s<r \in \mathbf{R}^{+}$,

$$
\begin{aligned}
\| 2 T_{f}(H \mid V ; r, s) \leq & \sum_{j=1}^{k} N\left(A_{j} ; r, s\right) \\
& +C\left(\log ^{+} T_{f}(r, s)\right)+o(\log r) .
\end{aligned}
$$

In particular, if $V=\mathbf{P}^{m}$, i.e., $f \in \mathscr{F}_{A}\left(\mathbf{C}^{n}, \mathbf{P}^{m}, m+3\right)$,

$$
\| 2 T_{f}(r, s) \leq \sum_{j=1}^{m+3} N(A ; r, s)+C\left(\log ^{+} T_{f}(r, s)\right)+o(\log r) \text {. }
$$

Proof. From [S4, Theorem 3.1] we have

$$
\begin{aligned}
\| 2 T_{f}(H \mid V ; r, s) \leq & \sum_{j=1}^{k} N_{f}\left(H_{j} ; r, s\right)-N\left(R_{f} ; r, s\right) \\
& +C\left(\log ^{+} T_{f}(r, s)\right)+o(\log r),
\end{aligned}
$$

where $R_{f}$ is the ramification divisor of $f$ which is defined in [S4, p. 73], or [D2]. By [D2, Lemma 3.2],

$$
\sum_{j=1}^{k} N_{f}\left(H_{j} ; r, s\right)-N\left(R_{f} ; r, s\right) \leq \sum_{j=1}^{k} \bar{N}_{f}\left(H_{j} ; r, s\right)+C
$$

where $\bar{N}_{f}(v ; r, s):=N\left(\left(f^{*} v\right)^{(1)} ; r, s\right)$ (cf. $\left.(2.2)\right)$. Note that $\bar{N}_{f}\left(H_{j} ; r, s\right)$ $=N\left(A_{j} ; r, s\right)$; then (4.1) is proved by (4.3) and (4.4).

LEMMA 4.2. Let $f \in \mathscr{F}_{B}\left(\mathbf{C}^{n}, \mathbf{P}^{m}, 3 m+1\right)$. Then for $0<s<r \in \mathbf{R}^{+}$,

$$
\| 2 T_{f}(r, s) \leq \sum_{j=1}^{3 m+1} N\left(A_{j} ; r, s\right)+C\left(\log ^{+} T_{f}(r, s)\right)+o(\log r) \text {. }
$$


Proof. From [V1, (5.5), (5.6), p. 103],

$$
\begin{array}{r}
{[(3 m+1)-m-1] T_{f}(r, s)=2 m T_{f}(r, s)} \\
\leq \log \left(\left|g_{1} \cdots g_{3 m+1}\right| / J\right)+\log D+C,
\end{array}
$$

where

(1) $g_{i}:=f_{0} a_{0}^{(i)}+\cdots+f_{m} a_{m}^{(i)}$, and $a_{0}^{(i)}, \ldots, a_{m}^{(i)}$ are given by (1.1), for $i=1, \ldots, 3 m+1$, and $\left(f_{0}, \ldots, f_{m}\right)$ is a reduced representation of $f$.

(2) $J:=\left\|f \wedge D^{\alpha_{1}} f \wedge \cdots \wedge D^{\alpha_{m}} f\right\|=\operatorname{det}\left(D^{\alpha_{l}} f\right)_{0 \leq i \leq m, \alpha_{0}=0}$, where $\left(0, \alpha_{1}, \ldots, \alpha_{m}\right)$ are nonnegative integers such that there exists a dense open subset $U$ of $\mathbf{C}^{n}$, for any $z \in U, f(z), D^{\alpha_{1}} f(z), \ldots, D^{\alpha_{m}} f(z)$ are linearly independent.

(3)

$$
D:=\operatorname{det}\left(\begin{array}{ccc}
1 & \cdots & 1 \\
D^{\alpha_{1}} f_{0} / f_{0} & \cdots & D^{\alpha_{1}} f_{m} / f_{m} \\
\cdots \cdots \cdots \cdots \cdots \cdots \cdots \cdots \cdots \\
D^{\alpha_{m}} f_{0} / f_{0} & \cdots & D^{\alpha_{m}} f_{m} / f_{m}
\end{array}\right) .
$$

From [F3, p. 255], we have

$$
\begin{aligned}
\int_{S(r)} & \log \left(\left|g_{1} \cdots g_{3 m+1}\right| / J\right) \sigma \\
\leq & \sum_{j=1}^{3 m+1} N_{f}^{H,}(r, s)^{\left[k_{0}\right]} \leq m \sum_{j=1}^{3 m+1} \bar{N}_{f}\left(H_{j} ; r, s\right),
\end{aligned}
$$

where

(1) By [F3, Proposition 4.5], $k_{0}$ always can be $m$ for nondegenerate meromorphic map from $\mathbf{C}^{n}$ into $\mathbf{P}^{m}$.

(2) By [F3, p. 250], the second inequality holds because

$$
\begin{aligned}
N_{f}^{H_{j}}(r, s)^{[m]} & :=N\left(\left(f^{*} H_{j}\right)^{(m)} ; r, s\right) \\
& \leq m \bar{N}_{f}\left(H_{j} ; r, s\right) .
\end{aligned}
$$

With (4.6), (4.7) and (4.8),

$$
2 m T_{f}(r, s) \leq \sum_{j=1}^{3 m+1} m \bar{N}_{f}\left(H_{j} ; r, s\right)+\int_{S(r)} \log D \cdot \sigma+C .
$$

Apply Lemma 3.1 repeatedly to $\int_{S(r)} \log D \cdot \sigma$, then (4.5) follows.

Lemma 4.3. For any $n, m \in \mathbf{Z}^{+}$, let $f, g: \mathbf{C}^{n} \rightarrow \mathbf{P}^{m}$ be linearly nondegenerate meromorphic maps with $f \neq \equiv$. Let $D$ be any hypersurface on $\mathbf{P}^{m}$. If $f^{-1}(D)=g^{-1}(D)$ and $f\left|f^{-1}(D)=g\right| g^{-1}(D)$, then

$$
\bar{N}_{f}(D ; r, s) \leq T_{f}(r, s)+T_{g}(r, s)+C \text {. }
$$


Proof. See [D2, Lemma 3.4]. (Note: Lemma 3.4 in [D2] needs the condition $n>m$, but this is seen to be unnecessary by checking the proof.)

LEMMA 4.4. Let $f, g \in \mathscr{F}_{B}\left(\mathbf{C}^{n}, \mathbf{P}^{m}, k\right)$ with $f \not \equiv g$ and with

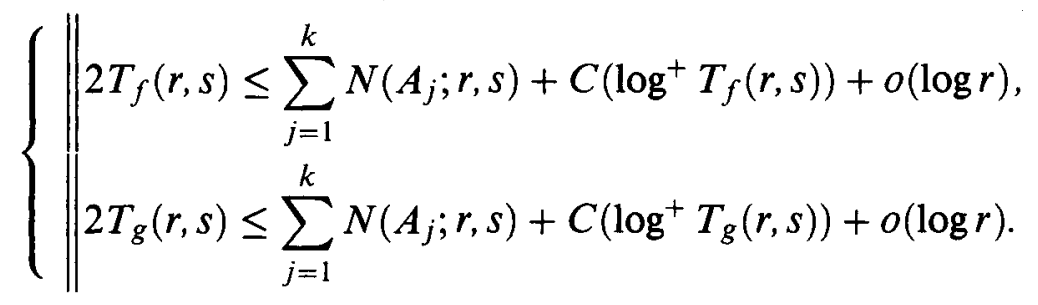

Then

$$
\| \lim _{r \rightarrow \infty} \sum_{j=1}^{k} N\left(A_{j} ; r, s\right) / T_{f}(r, s)=2 \text { and }
$$

$$
\| \lim _{r \rightarrow \infty} T_{f}(r, s) / T_{g}(r, s)=1 \text {. }
$$

Proof. From (4.10) and Lemma 4.3,

$$
\begin{aligned}
& \| T_{f}(r, s)+T_{g}(r, s) \leq \sum_{j=1}^{k} N\left(A_{j} ; r, s\right) \\
& \quad+C\left(\log ^{+} T_{f}(r, s)+\log T_{g}^{+}(r, s)\right)+o(\log r) \\
& \leq \\
& \quad T_{f}(r, s)+T_{g}(r, s)+C\left(\log ^{+} T_{f}(r, s)+\log ^{+} T_{g}(r, s)\right) \\
& \quad+o(\log r) .
\end{aligned}
$$

So,

(4.13)

$$
\begin{aligned}
\| 1 & \leq \frac{\left[\sum_{j=1}^{k} N\left(A_{j} ; r, s\right)+C\left(\log ^{+} T_{f}(r, s)+\log ^{+} T_{g}(r, s)\right)+o(\log r)\right]}{\left[T_{f}(r, s)+T_{g}(r, s)\right]} \\
& \leq 1+\frac{\left[C\left(\log ^{+} T_{f}(r, s)+\log ^{+} T_{g}(r, s)\right)+o(\log r)\right]}{\left[T_{f}(r, s)+T_{g}(r, s)\right]} .
\end{aligned}
$$

By (4.10),

$$
\| \varlimsup_{r \rightarrow \infty} T_{f}(r, s) / \sum_{j=1}^{k} N\left(A_{j} ; r, s\right) \leq 1 / 2 \text { and }
$$

$$
\| \varlimsup_{r \rightarrow \infty} T_{g}(r, s) / \sum_{j=1}^{k} N\left(A_{j} ; r, s\right) \leq 1 / 2 .
$$


Apply (4.15) to (4.13), we obtain (4.11). Then apply (4.11) to (4.13), we obtain (4.12).

Corollary 4.5. Let $f, g \in \mathscr{F}_{A}\left(\mathbf{C}^{n}, \mathbf{P}^{m}, m+3\right)$ (respectively $\mathscr{F}_{B}\left(\mathbf{C}^{n}, \mathbf{P}^{m}, 3 m+1\right)$, or $\left.\mathscr{F}_{C}\left(\mathbf{C}^{n}, V, k\right)\right)$ with $f \not \equiv g$. Then

$$
\| \lim _{r \rightarrow \infty} \sum_{j=1}^{m+3} N\left(A_{j} ; r, s\right) / T_{f}(r, s)=2 \text {, }
$$

(resp.

$$
\| \lim _{r \rightarrow \infty} \sum_{j=1}^{3 m+1} N\left(A_{j} ; r, s\right) / T_{f}(r, s)=2, \quad \text { or }
$$

$$
\begin{aligned}
& \left.\lim _{r \rightarrow \infty} \sum_{j=1}^{k} N\left(A_{j} ; r, s\right) / T_{f}(r, s)=2\right) \\
& \| \lim _{r \rightarrow \infty} T_{f}(r, s) / T_{g}(r, s)=1 .
\end{aligned}
$$

5. Proof for holomorphic curves. In this section, we prove Theorem $\mathrm{B}$ when $n=1$. Although this case is only for holomorphic curves, the method of proof will be essentially used for the remaining cases of Theorems A, B and C.

LEMMA 5.1. Let $n, m, k \in \mathbf{Z}^{+}$. Let $\mathscr{F}_{B}\left(\mathbf{C}^{n}, \mathbf{P}^{m}, k\right) \neq \varnothing$. Then there exists a dense subset $\mathscr{P} \subset \mathbf{C}_{*}^{m+1}$, such that for any $p=\left(p_{0}, \ldots, p_{m}\right) \in$ $\mathscr{P}$, the hyperplane $H_{p}$ defined by $p_{0} w_{0}+\cdots+p_{m} w_{m}=0$ satisfies

(5.1) $\operatorname{Codim}\left(\bigcup_{j=1}^{k} A_{j} \cap f^{-1}\left(H_{p}\right)\right) \geq 2$ for all $f \in \mathscr{F}_{B}\left(\mathbf{C}^{n}, \mathbf{P}^{m}, k\right)$.

Proof. For any irreducible pure $(n-1)$-dimensional component $\sigma$ of $\bigcup_{j=1}^{k} A_{j}$, set $K_{\sigma}:=\left\{\left(x_{0}, \ldots, x_{m}\right) \in \mathbf{C}^{m+1} \mid x_{0} f_{0}(z)+\cdots+x_{m} f_{m}(z)=0\right.$ for all $z \in \sigma\}$, where $\phi_{f}=\left(f_{0}, \ldots, f_{m}\right)$ is a reduced representation of $f \in \mathscr{F}_{B}\left(\mathbf{C}^{n}, \mathbf{P}^{m}, k\right) . K_{\sigma}$ is independent of the choice of $f$ for $f \mid A=$ $F \mid A . K_{\sigma}$ is a complex vector subspace of $\mathbf{C}^{m+1}$ with $\operatorname{dim} K_{\sigma} \leq m$.

Let $K:=\bigcup_{\sigma} K_{\sigma}$. Then $K$ is a union of at most a countable number of $m$-dimensional complex vector subspaces in $\mathbf{C}^{m+1}$. Let $\mathscr{P}:=$ $\mathbf{C}^{m+1}-K$. Then $\mathscr{P}$ meets the requirement of the lemma. 
Now for any $n, m, k \in \mathbf{Z}^{+}$and for $f, g, h \in \mathscr{F}_{B}\left(\mathbf{C}, \mathbf{P}^{m}, k\right)$, let $\mathscr{P}$ be associated to $\mathscr{F}_{B}\left(\mathbf{C}, \mathbf{P}^{m}, k\right)$ as in Lemma 5.1, and take $p=\left(p_{0}, \ldots, p_{m}\right) \in$ $\mathscr{P}$. Take $i \in\{1, \ldots, k\}$. We define a meromorphic function $\varphi$ on $\mathbf{C}$ by

$$
\begin{aligned}
\varphi & =\varphi(f, g, h, p, i):=\operatorname{det}\left(\begin{array}{ccc}
F & G & H \\
F^{\prime} / F & G^{\prime} / G & H^{\prime} / H \\
1 & 1 & 1
\end{array}\right) \\
& =\left(\frac{G^{\prime}}{G}-\frac{H^{\prime}}{H}\right) F+\left(\frac{H^{\prime}}{H}-\frac{F^{\prime}}{F}\right) G+\left(\frac{F^{\prime}}{F}-\frac{G^{\prime}}{G}\right) H,
\end{aligned}
$$

where

$$
\left\{\begin{array}{l}
F=F(f, p, i):=\left(a_{0}^{(i)} f_{0}+\cdots+a_{m}^{(i)} f_{m}\right) /\left(p_{0} f_{0}+\cdots+p_{m} f_{m}\right), \\
G=G(g, p, i):=\left(a_{0}^{(i)} g_{0}+\cdots+a_{m}^{(i)} g_{m}\right) /\left(p_{0} g_{0}+\cdots+p_{m} g_{m}\right), \\
H=H(h, p, i):=\left(a_{0}^{(i)} h_{0}+\cdots+a_{m}^{(i)} h_{m}\right) /\left(p_{0} h_{0}+\cdots+p_{m} h_{m}\right)
\end{array}\right.
$$

where $\phi_{f}=\left(f_{0}, \ldots, f_{m}\right), \phi_{g}=\left(g_{0}, \ldots, g_{m}\right), \phi_{h}=\left(h_{1}, \ldots, h_{m}\right)$ are reduced representations of $f, g, h$, respectively, and $\left(a_{0}^{(i)}, \ldots, a_{m}^{(i)}\right)$ is defined in (1.1).

Sometimes we define

$$
\begin{aligned}
P(f) & =p_{0} f_{0}+\cdots+p_{m} f_{m}, \\
H_{i}(f) & =a_{0}^{(i)} f_{0}+\cdots+a_{m}^{(i)} f_{m}, \text { etc. }
\end{aligned}
$$

Lemma 5.2. Let $f, g, h \in \mathscr{F}_{B}\left(\mathbf{C}, \mathbf{P}^{m}, 3 m+1\right)$. Assume

$$
\varphi=\varphi(f, g, h, p, i) \not \equiv 0,
$$

for some $p \in \mathscr{P}$ and some $i \in\{1, \ldots, 3 m+1\}$. Then for $0<s<r$,

$$
\| \lim _{r \rightarrow \infty} \frac{N\left(A_{i}, r, s\right)}{T_{f}(r, s)} \geq \frac{1}{2}
$$

Proof. First we prove

$$
\bigcup_{j=1, j \neq i}^{3 m+1} A_{j} \subset \varphi^{-1}(0) \cap\left(\varphi^{\prime}\right)^{-1}(0) .
$$

For any $a \in A_{j}$ with $j \neq i, j \in\{1, \ldots, 3 m+1\}$, because the $A_{k}$ are disjoint in $\mathbf{C}$ for $k=1, \ldots, 3 m+1$ and (5.1), we know that 
$F(a)=G(a)=H(a) \in \mathrm{C}_{*}$. Then from (5.2), we have

(5.7) $\varphi(a)=0$ and

(5.8) $\varphi^{\prime}(a)=\left(\frac{G^{\prime}(a)}{G(a)}-\frac{H^{\prime}(a)}{H(a)}\right) F^{\prime}(a)$

$$
\begin{aligned}
& +\left(\frac{H^{\prime}(a)}{H(a)}-\frac{F^{\prime}(a)}{F(a)}\right) G^{\prime}(a)+\left(\frac{F^{\prime}(a)}{F(a)}-\frac{G^{\prime}(a)}{G(a)}\right) H^{\prime}(a) \\
& +\left[\left(\frac{G^{\prime}}{G}\right)^{\prime}(a)-\left(\frac{H^{\prime}}{H}\right)^{\prime}(a)\right] F(a) \\
& +\left[\left(\frac{H^{\prime}}{H}\right)^{\prime}(a)-\left(\frac{F^{\prime}}{F}\right)^{\prime}(a)\right] G(a) \\
& +\left[\left(\frac{F^{\prime}}{F}\right)^{\prime}(a)-\left(\frac{G^{\prime}}{G}\right)^{\prime}(a)\right] H(a)=0 .
\end{aligned}
$$

Next we show that if $b \in A_{i}$, then

$$
\varphi(b) \neq \infty \text {. }
$$

When $w$ is near $b$ in $\mathbf{C}, F(w)=(w-b)^{k_{1}} F^{*}(w), F^{*}(b) \neq 0 ; G(w)=$ $(w-b)^{k_{2}} G^{*}(w), G^{*}(b) \neq 0 ; H(w)=(w-b)^{k_{3}} H^{*}(w), H^{*}(b) \neq 0$, where integers $k_{1}, k_{2}, k_{3} \geq 1$. Then $\varphi(b) \neq \infty$ follows from (5.2).

Since $\varphi \not \equiv 0$ and (5.6) and the First Main Theorem,

$$
\begin{aligned}
& \text { (5.10) } 2 \sum_{j=1, j \neq i}^{3 m+1} N\left(A_{j} ; r, s\right) \leq T_{\varphi}(r, s)+O(1)=N_{\varphi}(\infty ; r, s) \\
& +m_{\varphi}(\infty ; r)-m_{\varphi}(\infty ; s)+O(1) \text {. }
\end{aligned}
$$

By (5.2), (5.3) and (5.9), all the poles of $\varphi$ must be the ones of $F$, $G, H$. Hence

$$
N_{\varphi}(\infty ; r, s) \leq N_{F}(\infty ; r, s)+N_{G}(\infty ; r, s)+N_{H}(\infty ; r, s) .
$$

By Lemma 3.1,

$$
\begin{aligned}
\| m_{\varphi}(\infty ; r) \leq & m_{F}(\infty ; r)+m_{G}(\infty ; r)+m_{H}(\infty ; r) \\
& +C\left(\log ^{+} T_{F}(r, s)+\log ^{+} T_{G}(r, s)+\log ^{+} T_{H}(r, s)\right) \\
& +o(\log r) .
\end{aligned}
$$

Note $T_{F}(r, s) \leq T_{f}(r, s)+C$, etc. Note $f \not \equiv g$ because $\varphi \not \equiv 0$, so we can apply the Corollary 4.5. By (5.10), (5.11), (5.12) and (4.19),

$$
\| 2 \sum_{j=1, j \neq i}^{3 m+1} N\left(A_{j} ; r, s\right) \leq 3 T_{f}(r, s)+o(\log r)+C\left(\log ^{+} T_{f}(r, s)\right) .
$$

Then by (4.6) we prove (5.5). 
LeMmA 5.3. Let $\mathscr{F}_{B}\left(\mathbf{C}, \mathbf{P}^{m}, k\right), \mathscr{P}, p \in \mathscr{P}, i \in\{1, \ldots, k\}, f, g, h \in$ $\mathscr{F}_{B}\left(\mathbf{C}, \mathbf{P}^{m}, k\right), \varphi=\varphi(f, g, h, p, i), F=F(f, p, i), G=G(g, p, i), H=$ $H(h, p, i)$ be as in (5.2), (5.3). Assume $k \geq 2$. Then

$$
\varphi \equiv 0 \Leftrightarrow \text { There is constant }(\lambda, \mu, \nu) \in \mathbf{C}_{*}^{3} \text { such that }
$$

$$
\frac{\lambda}{F}+\frac{\mu}{G}+\frac{\nu}{H} \equiv 0
$$

Proof. Set $\alpha:=1 / F, \beta:=1 / G, \gamma:=1 / H$. Since

$$
\varphi=\operatorname{det}\left(\begin{array}{ccc}
1 / \alpha & 1 / \beta & 1 / \gamma \\
-\alpha^{\prime} / \alpha & -\beta^{\prime} / \beta & -\gamma^{\prime} / \gamma \\
1 & 1 & 1
\end{array}\right)=\frac{1}{\alpha \beta \gamma} \operatorname{det}\left(\begin{array}{ccc}
1 & 1 & 1 \\
\alpha & \beta & \gamma \\
\alpha^{\prime} & \beta^{\prime} & \gamma^{\prime}
\end{array}\right)
$$

and $\alpha \not \equiv \infty, \beta \not \equiv \infty, \gamma \not \equiv \infty$ for $f, g, h$ are linearly non-degenerate, put

$$
\Delta:=\operatorname{det}\left(\begin{array}{ccc}
1 & 1 & 1 \\
\alpha & \beta & \gamma \\
\alpha^{\prime} & \beta^{\prime} & \gamma^{\prime}
\end{array}\right)
$$

Thus (5.13) is equivalent to

$$
\Delta \equiv 0
$$

By Wronsky determinant, (5.14) is equivalent to

$$
\operatorname{det}\left(\begin{array}{ccc}
\alpha & \beta & \gamma \\
\alpha^{\prime} & \beta^{\prime} & \gamma^{\prime} \\
\alpha^{\prime \prime} & \beta^{\prime \prime} & \gamma^{\prime \prime}
\end{array}\right)=0
$$

It suffices to prove $(5.15) \Leftrightarrow(5.16)$.

For $(5.16) \Rightarrow(5.15)$ : By the definition of $\mathscr{F}_{B}\left(\mathbf{C}, \mathbf{P}^{m}, k\right)$ and $k \geq 2$, $H|A=G| A=H \mid A \not \equiv 0$, so $\lambda+\mu+\nu=0$ from (5.14). So (5.16) $\Leftrightarrow(5.14)$ $\Rightarrow(5.15)$ is obvious.

Assume (5.15). If $(\alpha, \beta, \gamma)=\zeta(1,1,1)$, where $\zeta$ is a meromorphic function, (5.16) is obvious; if not, then $\left(\alpha^{\prime}, \beta^{\prime}, \gamma^{\prime}\right)=\rho(1,1,1)+$ $\eta(\alpha, \beta, \gamma)$, where $\rho, \eta$ are meromorphic functions. Differentiating, we conclude that $\left(\alpha^{\prime \prime}, \beta^{\prime \prime}, \gamma^{\prime \prime}\right)$ is a linear combination of $(\alpha, \beta, \gamma)$ and $\left(\alpha^{\prime}, \beta^{\prime}, \gamma^{\prime}\right)$.

The proof of Theorem $\mathrm{B}$ when $n=1$.

(1) Let $f, g, h \in \mathscr{F}_{B}\left(\mathbf{C}, \mathbf{P}^{m}, 3 m+1\right)$. Let $\mathscr{P}$ be associated to $\mathscr{F}_{B}\left(\mathbf{C}, \mathbf{P}^{m}, 3 m+1\right)$ as in Lemma 5.1. 
It suffices to show that for each $p \in \mathscr{P}$, for all but at most four $i \in\{1, \ldots, 3 m+1\}$ (here the condition $m \geq 2$ is used),

$$
\frac{\lambda_{i}}{F(f, p, i)}+\frac{\mu_{i}}{G(g, p, i)}+\frac{\nu_{i}}{H(h, p, i)} \equiv 0
$$

holds for some constant $\left(\lambda_{i}, \mu_{i}, \nu_{i}\right) \in \mathbf{C}_{*}^{3}$, where $F(f, p, i), G(g, p, i)$, $H(h, p, i)$ are defined as in (5.3).

In fact, assume that the statement above has been proved, we prove that $f, g, h$ satisfy Property $(\mathrm{P})$ as follows: for any $p=\left(p_{0}, \ldots, p_{m}\right) \in$ $\mathbf{C}_{*}^{m+1}$, since $\mathscr{P}$ is dense in $\mathbf{C}_{*}^{m+1}$ by Lemma 5.1 , we choose a sequence of $p_{t} \in \mathscr{P}$ with $p_{t} \rightarrow p$ as $t \rightarrow \infty$. For each $p_{t}$, by the assumption, for all but at most four $i \in\{1, \ldots, 3 m+1\}$ (the exceptional set depends on $t$ ),

$$
\frac{\lambda_{t_{1}}}{F\left(f, p_{t}, i\right)}+\frac{\mu_{t_{i}}}{G\left(g, p_{t}, i\right)}+\frac{\nu_{t_{i}}}{H\left(h, p_{t}, i\right)} \equiv 0
$$

for some constant $\left(\lambda_{t_{i}}, \mu_{t_{i}}, \nu_{t_{i}}\right) \in \mathbf{C}_{*}^{3}$. We assume

$$
\operatorname{Max}\left(\left|\lambda_{t_{1}}\right|,\left|\mu_{t_{i}}\right|,\left|\nu_{t_{i}}\right|\right)=1 \text { for all } t \text { and } i \text {. }
$$

By taking a convergent subsequence of $\left\{p_{t}\right\}$ if necessary, we can assume that

(i) There is an exceptional set $\Delta_{p} \subset\{1, \ldots, 3 m+1\}$ which consists of at most four numbers and is independent of $t$, such that for any $p_{t}$, and any $i \in\{1, \ldots, 3 m+1\}-\Delta_{p},(5.18)$ holds for some constant $\left(\lambda_{t_{1}}, \mu_{t_{1}}, \nu_{t_{l}}\right) \in \mathbf{C}_{*}^{3}$.

(ii) $\lambda_{t_{i}} \rightarrow \lambda_{i}, \mu_{t_{i}} \rightarrow \mu_{i}, \nu_{t_{i}} \rightarrow \nu_{i}$ for each $i \in\{1, \ldots, 3 m+1\}-\Delta_{p}$ as $t \rightarrow \infty$.

Because (5.19), $\left(\lambda_{i}, \mu_{i}, \nu_{i}\right) \in \mathbf{C}_{*}^{3}$. Just let $t \rightarrow \infty$ in (5.18), we have proved (1.4), i.e., $f, g, h$ satisfy Property (P).

(2) Now suppose that the statement $(5.17)$ is not true. Then there exist $p \in \mathscr{P}$ and $i_{1}, \ldots, i_{5} \in\{1, \ldots, 3 m+1\}$, such that there is no constant $(\lambda, \mu, \nu) \in \mathbf{C}_{*}^{3}$ so that

$$
\frac{\lambda}{F\left(f, p, i_{s}\right)}+\frac{\mu}{G\left(g, p, i_{s}\right)}+\frac{\nu}{H\left(h, p, i_{s}\right)} \equiv 0
$$

for $s=1,2,3,4,5$. By Lemma 5.3, $\varphi\left(f, g, h, p, i_{s}\right) \not \equiv 0$ for $s=$ $1,2,3,4,5$. Then by Lemma 5.2 ,

$$
\| \lim _{r \rightarrow \infty} N\left(A_{i_{s}} ; r, s\right) / T_{f}(r, s) \geq \frac{1}{2}, \quad \text { for } s=1,2,3,4,5 .
$$


So,

$$
\begin{aligned}
\| \lim _{r \rightarrow \infty} & \sum_{j=1}^{3 m+1} N\left(A_{j} ; r, s\right) / T_{f}(r, s) \\
& \geq \sum_{j=1}^{3 m+1} \underset{r \rightarrow \infty}{\lim } N\left(A_{j}, r, s\right) / T_{f}(r, s) \geq \frac{5}{2}>2,
\end{aligned}
$$

but this is contrary to (4.17).

6. Lemmas for $\varphi_{a e}$. For proving Theorems A, B and C, we make some preparation in this section. Since $\S 5$, we assume $n \geq 2$ through $\S 6, \S 7$.

Let $n, m, k \in \mathbf{Z}^{+}$with $k \geq 2$, and let $f, g, h \in \mathscr{F}_{B}\left(\mathbf{C}^{n}, \mathbf{P}^{m}, k\right)$. Let $\mathscr{P}$ be associated to $\mathscr{F}_{B}\left(\mathbf{C}^{n}, \mathbf{P}^{m}, k\right)$ as in Lemma 5.1. Take $p \in \mathscr{P}$ and $i \in\{1, \ldots, k\}$. Suppose that there is no constant $(\lambda, \mu, \nu) \in \mathbf{C}_{*}^{3}$, such that

$$
\frac{\lambda}{F}+\frac{\mu}{G}+\frac{\nu}{H} \equiv 0,
$$

where $F=F(f, p, i), G=G(g, p, i), H=H(h, p, i)$ are defined by (5.3).

\section{Let}

$$
\begin{aligned}
K:= & K(f, g, h, p, i):=\{F=0\} \cup\{1 / F=0\} \cup\{G=0\} \cup\{1 / G=0\} \\
& \cup\{H=0\} \cup\{1 / H=0\} \cup\{F=G\} \cup\{G=H\} \cup\{H=F\} .
\end{aligned}
$$

Since $f, g, h$ are linearly non-degenerate and the assumption in (6.1), $K$ is a proper analytic subset of $\mathbf{C}^{n}$.

Take $a=\left(a_{1}, \ldots, a_{n}\right) \in \mathbf{C}^{n}-K$. Since $n \geq 2$, let $e=\left(e_{1}, \ldots, e_{n}\right) \in$ $E:=\{1\} \times \mathbf{C}^{n-1}($ see $\S 3)$. Then define a meromorphic function $\varphi_{a e}=$ $\varphi_{a e}(f, g, h, p, i)$ on $\mathbf{C}$ by

$$
\varphi_{a e}:=\operatorname{det}\left(\begin{array}{ccc}
F_{a e} & G_{a e} & H_{a e} \\
F_{a e}^{\prime} / F_{a e} & G_{a e}^{\prime} / G_{a e} & H_{a e}^{\prime} / H_{a e} \\
1 & 1 & 1
\end{array}\right) \text {, where }
$$

$$
F_{a e}(w):=F(a+w e) \text {, etc. }
$$

LEMMA 6.1.

$$
\varphi_{a e}(0)=A_{1}(a)+A_{2}(a) e_{2}+\cdots+A_{n}(a) e_{n},
$$


where

$$
\begin{aligned}
A_{j}(a)= & \left(\frac{D^{j} G(a)}{G(a)}-\frac{D^{j} H(a)}{H(a)}\right) F(a)+\left(\frac{D^{j} H(a)}{H(a)}-\frac{D^{j} F(a)}{F(a)}\right) G(a) \\
& +\left(\frac{D^{j} F(a)}{F(a)}-\frac{D^{j} G(a)}{G(a)}\right) H(a) \neq \infty \\
& \text { for } j=1, \ldots, n \text { and } D^{j}=\frac{\partial}{\partial z^{j}} .
\end{aligned}
$$

Proof. By (6.3), $F_{a e}(0)=F(a)$, and

$$
F_{a e}^{\prime}(w)=D^{1} F(a+w e)+e_{2} D^{2} F(a+w e)+\cdots+e_{n} D^{n} F(a+w e) \text {, }
$$

so

$$
F_{a e}^{\prime}(0)=D^{1} F_{a e}(0)+e_{2} D^{2} F_{a e}(0)+\cdots+e_{n} D^{n} F_{a e}(0) .
$$

Then by (6.2) and (6.5), we obtain (6.4).

Since $a \notin K, F(a) \neq 0, \infty, G(a) \neq 0, \infty, H(a) \neq 0, \infty$, so $A_{j}(a) \neq \infty$ for $j=1, \ldots, n$.

LEMMA 6.2. There is no open subset $U \subset \mathbf{C}^{n}-K$ such that

$$
\varphi_{a e}(0)=0 \text { for all } a \in U \text { and all } e \in E \text {. }
$$

Proof. (1) Suppose that there is some open subset $U \subset \mathbf{C}^{n}-K$ such that $\varphi_{a e}(0)=0$ for all $a \in U$ and all $e \in E$. From Lemma 6.1, it implies that $A_{j}(a)=0$ for all $a \in U$ and for $j=1, \ldots, n$. Since $A_{j}$ are meromorphic, we know

$$
A_{j} \equiv 0 \quad \text { on } \mathbf{C}^{n} \quad \text { for } j=1, \ldots, n \text {. }
$$

We shall find a contradiction with (6.7).

(2) Consider $A_{n} \equiv 0$. Write $z=\left(z^{\prime}, z_{n}\right) \in \mathbf{C}^{n-1} \times \mathbf{C}$. $A_{n} \equiv 0$ means

$$
\operatorname{det}\left(\begin{array}{ccc}
F\left(z^{\prime}, z_{n}\right) & G\left(z^{\prime}, z_{n}\right) & H\left(z^{\prime}, z_{n}\right) \\
\frac{D^{n} F\left(z^{\prime}, z_{n}\right)}{F\left(z^{\prime}, z_{n}\right)} & \frac{D^{n} G\left(z^{\prime}, z_{n}\right)}{G\left(z^{\prime}, z_{n}\right)} & \frac{D^{n} H\left(z^{\prime}, z_{n}\right)}{H\left(z^{\prime}, z_{n}\right)} \\
1 & 1 & 1
\end{array}\right)=0
$$

for all $\left(z^{\prime}, z_{n}\right) \in \mathbf{C}^{n}$.

For any $\left(z^{\prime}, z_{n}\right) \in \mathbf{C}^{n}-K$, fixing $z^{\prime} \in \mathbf{C}^{n-1}$, by the proof of Lemma 5.3 for $F\left(z^{\prime}, \cdot\right) \not \equiv 0, G\left(z^{\prime}, \cdot\right) \not \equiv 0, H\left(z^{\prime}, \cdot\right) \not \equiv 0$, there is constant $\left(\tilde{\lambda}\left(z^{\prime}\right), \tilde{\mu}\left(z^{\prime}\right), \tilde{\nu}\left(z^{\prime}\right)\right) \in \mathbf{C}_{*}^{3}$ such that

$$
\left\{\begin{array}{l}
\frac{\tilde{\lambda}\left(z^{\prime}\right)}{F\left(z^{\prime}, z_{n}\right)}+\frac{\tilde{\mu}\left(z^{\prime}\right)}{G\left(z^{\prime}, z_{n}\right)}+\frac{\tilde{\nu}\left(z^{\prime}\right)}{H\left(z^{\prime}, z_{n}\right)} \equiv 0, \\
\tilde{\lambda}\left(z^{\prime}\right)+\tilde{\mu}\left(z^{\prime}\right)+\tilde{\nu}\left(z^{\prime}\right) \equiv 0, \quad \text { for all } z_{n} \in \mathbf{C} .
\end{array}\right.
$$


The second identity is because $F|A=G| A=H \mid A \not \equiv 0$ for $k \geq 2$.

(3) Consider the equation on $\mathbf{C}^{n}$,

$$
\left\{\begin{array}{l}
\frac{\lambda(z)}{F(z)}+\frac{\mu(z)}{G(z)}+\frac{\nu(z)}{H(z)} \equiv 0, \\
\lambda(z)+\mu(z)+\nu(z) \equiv 0 .
\end{array}\right.
$$

For any $z \in \mathbf{C}^{n}-K$, we solve (6.9) to obtain

$$
\left\{\begin{array}{l}
\lambda(z)=\frac{1 / H(z)-1 / G(z)}{1 / F(z)-1 / G(z)} \nu(z), \\
\mu(z)=\frac{1 / F(z)-1 / H(z)}{1 / F(z)-1 / G(z)} \nu(z) .
\end{array}\right.
$$

So, for any $\nu \not \equiv 0$, we obtain a solution $(\lambda, \mu, \nu)$ for $(6.9)$ on $\mathbf{C}^{n}-K$, with $\mu \not \equiv 0$ and $\lambda \not \equiv 0$. If we fix $\nu \not \equiv 0$ to be a meromorphic function, then $(\lambda(z), \mu(z), \nu(z))$ is a solution for (6.9) on all of $\mathbf{C}^{n}$, where $\lambda \not \equiv 0$, $\mu \not \equiv 0$ are also meromorphic functions.

Such $(\lambda, \mu, \nu)$ is not unique, but for any $z \in \mathbf{C}^{n}-K$,

$$
\frac{\lambda(z)}{\nu(z)} \neq 0, \infty, \quad \frac{\mu(z)}{\nu(z)} \neq 0, \infty, \quad \frac{\lambda(z)}{\mu(z)} \neq 0, \infty
$$

are uniquely determined by (6.9) because of (6.10).

We want to prove that such $\lambda / \nu, \mu / \nu, \lambda / \mu$ are constant on $\mathbf{C}^{n}$ from (6.7), which is contrary to (6.1). Thus the lemma is proved.

(4) For any $z=\left(z^{\prime}, z_{n}\right) \in \mathbf{C}^{n}-K$, by (6.8) and the uniqueness property in (3), assume $\tilde{\nu}\left(z^{\prime}\right) \neq 0$; then we have

$$
\frac{\tilde{\lambda}\left(z^{\prime}\right)}{\tilde{\nu}\left(z^{\prime}\right)}=\frac{\lambda\left(z^{\prime}, z_{n}\right)}{\nu\left(z^{\prime}, z_{n}\right)} \neq 0, \infty, \quad \frac{\tilde{\mu}\left(z^{\prime}\right)}{\tilde{\nu}\left(z^{\prime}\right)}=\frac{\mu\left(z^{\prime}, z_{n}\right)}{\nu\left(z^{\prime}, z_{n}\right)} \neq 0, \infty,
$$

Thus

$$
\frac{\tilde{\lambda}\left(z^{\prime}\right)}{\tilde{\mu}\left(z^{\prime}\right)}=\frac{\lambda\left(z^{\prime}, z_{n}\right)}{\mu\left(z^{\prime}, z_{n}\right)} \neq 0, \infty .
$$

i.e., for any $\left(z^{\prime}, z_{n}\right) \in \mathbf{C}^{n}-K$, the meromorphic functions $\lambda / \mu, \nu / \mu$, $\lambda / \nu$ are independent of $z_{n}$. So, they are independent of $z_{n}$ on $\mathbf{C}^{n}$.

Repeating the same procedure for $j=1, \ldots, n-1$, we prove that $\lambda / \mu, \nu / \mu, \lambda / \nu$ are independent of $z_{j}$ for $j=1, \ldots, n$.

7. Proof for Theorems A, B, C. As in $\S 6$, assume that $n \geq 2$.

LEMMA 7.1. Let $f, g, h \in \mathscr{F}_{A}\left(\mathbf{C}^{n}, \mathbf{P}^{m}, m+3\right)$ (respectively $\mathscr{F}_{B}\left(\mathbf{C}^{n}, \mathbf{P}^{m}, 3 m+1\right)$, or $\left.\mathscr{F}_{C}\left(\mathbf{C}^{n}, V, k\right)\right)$. Let $p \in \mathscr{P}$, where $\mathscr{P}$ is associated to $\mathscr{F}_{A}\left(\mathbf{C}^{n}, \mathbf{P}^{m}, m+3\right)\left(\right.$ resp. $\mathscr{F}_{B}\left(\mathbf{C}^{n}, \mathbf{P}^{m}, 3 m+1\right)$, or $\left.\mathscr{F}_{C}\left(\mathbf{C}^{n}, V, k\right)\right)$ 
and let $i \in\{1, \ldots, m+3\}$ (resp. $\{1, \ldots, 3 m+1\}$, or $\{1, \ldots, k\})$. Let $F=F(f, p, i), G=G(g, p, i), H=H(h, p, i)$ be defined by (5.3). Suppose that there is no constant $(\lambda, \mu, \nu) \in \mathbf{C}_{*}^{3}$ such that

$$
\frac{\lambda}{F}+\frac{\mu}{G}+\frac{\nu}{H} \equiv 0 \text {. }
$$

For each $b \in \mathbf{C}^{n}$, define a bioholomorphic map $\zeta_{b}: \mathbf{C}^{n} \rightarrow \mathbf{C}^{n}$ by $\zeta_{b}(z)=$ $z+b$ for all $z \in \mathbf{C}^{n}$. Abbreviate $A_{j b}=\zeta_{b}^{-1}\left(A_{j}\right)$ and $f_{b}=f \circ \zeta_{b}$. Then for almost every $b \in \mathbf{C}^{n}$ we have

$$
\| \varliminf_{r \rightarrow \infty} N\left(A_{j b} ; r, s\right) / T_{f_{b}}(r, s) \geq \frac{1}{2}, \quad \text { for } 0<s<r .
$$

Proof. We only need to consider $\mathscr{F}_{A}\left(\mathbf{C}^{n}, \mathbf{P}^{m}, m+3\right)$. For the cases of $\mathscr{F}_{B}\left(\mathbf{C}^{n}, \mathbf{P}^{m}, 3 m+1\right)$, or $\mathscr{F}_{C}\left(\mathbf{C}^{n}, V, k\right)$, the proof is the same.

(1) By $\S 6$, there is a proper analytic subset $K:=K(f, g, h, p, i) \in \mathbf{C}^{n}$ and $a \in \mathbf{C}^{n}-K$ and some $e \in E$ such that

$$
\varphi_{a e}(0) \neq 0,
$$

where $\varphi_{a e}=\varphi_{a e}(f, g, h, p, i)$ is defined by (6.2).

By taking $w=\left(w_{1}, \ldots, w_{n}\right)=a+\left(z_{1}, \ldots, z_{n}\right)=a+z$, we assume $a=0$. From Lemma $6.1, E_{0}:=\left\{e \in E \mid \varphi_{0 e}(0)=0\right\} \subset E$ is a proper linear variety, so

$$
\text { Measure } E_{0}=0 \text {. }
$$

(2) For any $e \in E-E_{0}$ and for any $j \in\{1, \ldots, m+3\}$ with $j \neq i$, we shall prove

$$
\begin{gathered}
I_{e j} \subset \varphi_{0 e}^{-1}(0) \cap\left(\varphi_{0 e}^{\prime}\right)^{-1}(0), \text { where } \\
I_{e j}:=\left\{w \in \mathbf{C} \mid w e \in A_{j}-f^{-1}\left(H_{p}\right) \cup \underset{\substack{1 \leq u \leq m+3 \\
u \neq j}}{\bigcup} A_{u}\right\} .
\end{gathered}
$$

Assume $I_{e j} \neq \varnothing$. For any $b \in I_{e j}$, since $F_{0 e}(b)=F(b e)=G_{0 e}(b)=$ $G(b e)=H_{0 e}(b)=H(b e) \in \mathbf{C}_{*}$ by definition (7.6), by using the proof of Lemma $5.2((5.7),(5.8))$, we can prove

$$
\varphi_{0 e}(b)=\varphi_{0 e}^{\prime}(b)=0 \text {. }
$$

So, (7.5) is proved.

(3) Now we estimate $2 \sum_{j=1, j \neq i}^{m+3} N\left(A_{j} ; r, s\right)$. 
Because $p \in \mathscr{P}$ and $\operatorname{Codim} A_{i} \cap A_{j} \geq 2$ for $i \neq j$, we know that

$$
\begin{gathered}
\operatorname{Codim} A_{j} \cap\left\{f^{-1}\left(H_{p}\right) \cup \bigcup_{\substack{1 \leq u \leq m+3 \\
u \neq j}} A_{u}\right\} \geq 2, \text { so } \\
N\left(A_{j} ; r, s\right)=\int_{s}^{r} \frac{d t}{t^{2 n-1}} \int_{A_{j}^{\prime} \cap B(t)} \varphi_{0}^{n-1}
\end{gathered}
$$

where

$$
A_{j}^{\prime}=A_{j}-f^{-1}\left(H_{p}\right) \cup \bigcup_{\substack{1 \leq u \leq m+3 \\ u \neq j}} A_{u}
$$

Therefore

$$
\begin{aligned}
& 2 \sum_{j=1, j \neq i}^{m+3} N\left(A_{j} ; r, s\right) \\
& \quad=2 \int_{e \in E-E_{0}} \sum_{j=1, j \neq i}^{m+3} N\left(I_{e j} ; \frac{r}{|e|}, \frac{s}{|e|}\right) L^{*}\left(\omega_{0}^{n-1}\right)(e) \\
& \quad\left(\begin{array}{l}
\because 7.4) \text { and } L^{*} \\
\text { is defined in }(3.3)
\end{array}\right) \\
& \quad \leq \int_{e \in E-E_{0}} N_{\varphi_{0 e}}\left(0 ; \frac{r}{|e|}, \frac{s}{|e|}\right) L^{*}\left(\omega_{0}^{n-1}\right)(e) .
\end{aligned}
$$

The last inequality is because of (7.5) and the fact that if $I_{e j}=\varnothing$, then $N\left(I_{e j} ; r, s\right)=0$.

(4) For any $e \in E-E_{0}$, we estimate $N_{\varphi_{0 c}}(0 ; r /|e|, s /|e|)$ now.

If $\varphi_{0 e} \equiv$ const., since $\varphi_{0 e}(0) \neq 0, N_{\varphi_{0 e}}(0 ; r /|e|, s /|e|) \equiv 0$. So, we consider $\varphi_{0 e} \not \equiv$ const. Then by the First Main Theorem,

$$
\begin{aligned}
& N_{\varphi_{0 e}}\left(0 ; \frac{r}{|e|}, \frac{s}{|e|}\right) \leq T_{\varphi_{0 e}}\left(\frac{r}{|e|}, \frac{s}{|e|}\right)+m_{\varphi_{0 e}}\left(0 ; \frac{s}{|e|}\right)+C \\
& \quad \leq N_{\varphi_{0 e}}\left(\infty ; \frac{r}{|e|}, \frac{s}{|e|}\right)+m_{\varphi_{0 e}}\left(\infty ; \frac{r}{|e|}\right)+m_{\varphi_{0 e}}\left(0 ; \frac{s}{|e|}\right)+C .
\end{aligned}
$$

By (6.2) and as in (5.9), since $\varphi_{0 e} \not \equiv$ const., all the poles of $\varphi_{0 e}$ must be the ones of $F_{0 e}, G_{0 e}$, and $H_{0 e}$. Then

$$
\begin{aligned}
N_{\varphi_{0 e}}\left(\infty ; \frac{r}{|e|}, \frac{s}{|e|}\right) \leq & N_{F_{0 e}}\left(\infty ; \frac{r}{|e|}, \frac{s}{|e|}\right) \\
& +N_{G_{0 e}}\left(\infty ; \frac{r}{|e|}, \frac{s}{|e|}\right)+N_{H_{0 e}}\left(\infty ; \frac{r}{|e|}, \frac{s}{|e|}\right) .
\end{aligned}
$$


Furthermore,

$$
\begin{aligned}
m_{\varphi_{0 e}} & \left(\infty ; \frac{t}{|e|}\right) \\
\leq & m_{F_{0 e}}\left(\infty ; \frac{t}{|e|}\right)+m_{G_{0 e}}\left(\infty ; \frac{t}{|e|}\right)+m_{H_{0 e}}\left(\infty ; \frac{t}{|e|}\right) \\
& +2\left(m_{F_{0 e}^{\prime} / F_{0 e}}\left(\infty ; \frac{t}{|e|}\right)+m_{G_{0 e}^{\prime} / G_{0 e}}\left(\infty ; \frac{t}{|e|}\right)+m_{H_{0 e}^{\prime} / H_{0 e}}\left(\infty ; \frac{t}{|e|}\right)\right) \\
& +\log 6
\end{aligned}
$$

So,

$$
\begin{aligned}
& N_{\varphi_{0 e}}\left(0 ; \frac{r}{|e|}, \frac{s}{|e|}\right) \\
& \leq T_{F_{0 e}}\left(\frac{r}{|e|}, \frac{s}{|e|}\right)+T_{G_{0 e}}\left(\frac{r}{|e|}, \frac{s}{|e|}\right)+T_{H_{0 e}}\left(\frac{r}{|e|}, \frac{s}{|e|}\right) \\
& \quad+2\left(m_{F_{0 e}^{\prime} / F_{0 e}}\left(\infty ; \frac{r}{|e|}\right)+m_{G_{0 e}^{\prime} / G_{0 e}}\left(\infty ; \frac{r}{|e|}\right)\right. \\
& \left.\quad+m_{H_{0 e}^{\prime} / H_{0 e}}\left(\infty ; \frac{r}{|e|}\right)\right)+\log 6 .
\end{aligned}
$$

(5) By (3), (4), and Lemma 3.2,

$$
\begin{aligned}
& \| 2 \sum_{j=1, j \neq i}^{m+3} N\left(A_{j} ; r, s\right) \\
& \leq \int_{e \in E-E_{0}} N_{\varphi_{0 e}}\left(0 ; \frac{r}{|e|}, \frac{s}{|e|}\right) L^{*}\left(\omega_{0}^{n-1}\right)(e) \\
& \leq T_{F}(r, s)+T_{G}(r, s)+T_{H}(r, s) \\
& \quad+C\left(\log T_{F}^{+}(r, s)+\log ^{+} T_{G}(r, s)+\log ^{+} T_{H}(r, s)\right) \\
& \quad+o(\log r)+C .
\end{aligned}
$$

By Corollary 4.5, and (7.7), the lemma for $\mathscr{F}_{A}\left(\mathbf{C}^{n}, \mathbf{P}^{m}, m+3\right)$ follows as in the proof of Lemma 5.2.

REMARK. If $f, g, h, p$ are as in Lemma 7.1, suppose for distinct $i_{t} \in\{1, \ldots, m+3\}$ (resp. $\{1, \ldots, 3 m+1\}$, or $\{1, \ldots, k\}$ ), there are no constant $\left(\lambda_{t}, \mu_{t}, \nu_{t}\right) \in \mathbf{C}_{*}^{3}$, such that

$$
\frac{\lambda_{t}}{F\left(f, p, i_{t}\right)}+\frac{\mu_{t}}{G\left(g, p, i_{t}\right)}+\frac{\nu_{t}}{H\left(h, p, i_{t}\right)} \equiv 0, \quad \text { for } t=1, \ldots, 5 .
$$


Then there is $a \in \mathbf{C}^{n}$, which is independent of $t$, so that under the new coordinates system $w=a+z$,

$$
\| \lim _{r \rightarrow \infty} N\left(A_{i} ; r, s\right) / T_{f}(r, s) \geq \frac{1}{2} .
$$

In fact, from $\S 6$, we first consider $i_{1}$, there is $a_{1} \in \mathbf{C}^{n}$, and some $e_{1} \in E, \varphi_{a_{1} e_{1}}(0) \neq 0$, where $\varphi_{a_{1} e_{1}}=\varphi_{a_{1} e_{1}}\left(f, g, h, p, i_{1}\right)$. By Lemma 6.1, there are neighborhoods $U_{1}$ of $a_{1}$ in $\mathbf{C}^{n}$ and $V_{1}$ of $e_{1}$ in $E$, so that for all $a \in U_{1}, e \in V_{1}, \varphi_{a e}(0) \neq 0$. Then, we consider $i_{2}$, also from $\S 6$, there is $a_{2} \in U_{1}$ and some $e_{2} \in V_{1}, \varphi_{a_{2} e_{2}}(0) \neq 0$, and then go on for $t=3,4,5$. So, we finally find some $a \in \mathbf{C}^{n}$, and $e \in E$, such that $\varphi_{a e}(f, g, h, p, i)(0) \neq 0$, for $t=1,2,3,4,5$.

Proof of Theorems A, B and C. From Lemma 7.2 and the remark above and as the proof of Theorem $\mathrm{B}$ when $n=1$ in $\S 5$, Theorems $\mathrm{A}$ and $\mathrm{B}$ are proved immediately.

For Theorem $\mathrm{C}$, for $f, g, h \in \mathscr{F}_{C}\left(\mathbf{C}^{n}, V, k\right)$, we can prove as above that $f, g, h$ satisfy Property (P). It remains to show that $f, g, h$ are algebraically dependent.

Since $f, g, h$ satisfy Property (P) and $k \geq 5$, we can take $p=$ $\left(p_{0}, \ldots, p_{m}\right) \in \mathbf{C}_{*}^{m+1}$ and $i \in\{1, \ldots, k\}$ such that

$$
\left(a_{0}^{(i)} w_{0}+\cdots+a_{M}^{(i)} w_{M}\right) /\left(p_{0} w_{0}+\cdots+p_{M} w_{M}\right) \not \equiv \text { const. } \quad \text { and }
$$

$$
\frac{\lambda P(f)}{H_{i}(f)}+\frac{\mu P(g)}{H_{i}(g)}+\frac{\nu P(h)}{H_{i}(h)} \equiv 0
$$

for some $(\lambda, \mu, \nu) \in \mathbf{C}_{*}^{3}$, where $P(f)=p_{0} f_{0}+\cdots+p_{M} f_{M}, H_{i}(f)=$ $a_{0}^{(i)} f_{0}+\cdots+a_{M}^{(i)} f_{M}$, etc., as in (5.4).

Let

$$
\begin{aligned}
Q(w, u, w):= & \lambda P(w) H_{i}(u) H_{i}(v)+\mu H_{i}(w) P(u) H_{i}(v) \\
& +\nu H_{i}(w) H_{i}(u) P(v)
\end{aligned}
$$

be a polynomial of $\mathbf{C}\left[w_{0}, \ldots, w_{M} ; u_{0}, \ldots, u_{M} ; v_{0}, \ldots, v_{M}\right]$. Note that

(7.10) $Q(w, u, v)=H_{i}(w) H_{i}(u) H_{i}(v)\left(\frac{\lambda P(w)}{H_{i}(w)}+\frac{\mu P(u)}{H_{i}(u)}+\frac{\nu P(v)}{H_{i}(v)}\right)$.

Assume $\lambda \neq 0$. Since $V$ is not contained in any hyperplane in $\mathbf{P}^{M}$, we can choose $u^{\prime}, v^{\prime} \in \mathbf{C}^{M+1}$ with $\mathbf{P}\left(u^{\prime}\right) \in V$ and $\mathbf{P}\left(v^{\prime}\right) \in V$, such that $H_{i}\left(u^{\prime}\right) \neq 0, H_{i}\left(v^{\prime}\right) \neq 0$. Hence $Q\left(\cdot, u^{\prime}, v^{\prime}\right) \not \equiv 0$ by (7.10). For the same reason as above we can choose $w^{\prime} \in \mathbf{C}^{M+1}$ with $\mathbf{P}\left(w^{\prime}\right) \in V$ such 
that $Q\left(w^{\prime}, u^{\prime}, w^{\prime}\right) \neq 0$. This means $\{(\mathbf{P}(w), \mathbf{P}(u), \mathbf{P}(v)) \in V \times V \times$ $V \mid Q(w, u, v)=0\}$ is a proper analytic subset of $V \times V \times V$. Then from (7.9), $Q\left(\phi_{f}, \phi_{g}, \phi_{h}\right) \equiv 0$, where $\phi_{f}$ is a reduced representation of $f$, etc., and therefore $f, g, h$ are algebraically dependent.

REMARK. Recently, Stoll has proved some closely related results [S11] which implies that if $f, g, h \in \mathscr{F}_{A}\left(\mathbf{C}^{n}, \mathbf{P}^{m}, m+3\right)$, or $\mathscr{F}_{B}\left(\mathbf{C}^{n}, \mathbf{P}^{m}, 3 m+1\right)$, or $\mathscr{F}_{C}\left(\mathbf{C}^{n}, V, k\right)$, then $f, g, h$ are not in general position, hence $f, g, h$ are algebraically dependent. Stoll applies the First Main Theorem for exterior product [S10] to prove his theorems. The method is very interesting.

\section{REFERENCES}

[C1] H. Cartan, Sur quelques théorèmes de M. R. Nevanlinna, C. R. Acad. Sci. Paris, 185 (1927), 1253-1254.

[C2] Un nouveau théorème d'unicité relatif aux fonctions méromorphes, C. R. Acad. Sci. Paris, 188 (1929), 301-303.

[D1] S. J. Drouilhet, Ramification and unicity of equidimensional holomorphic maps, thesis, Rice University, 1974.

[D2] _ A unicity theorem for meromorphic mappings between algebraic varieties, Trans. Amer. Math. Soc., 265 (1981), 349-358.

[F1] $\mathrm{H}$. Fujimoto, The uniqueness problem of meromorphic maps into the complex projective space, Nagoya Math. J., 58 (1975), 1-23.

[F2] Remarks to the uniqueness problem of meromorphic maps into $\mathbf{P}^{N}(\mathbf{C})$, III, Nagoya Math. J., 75 (1979), 71-85.

[F3] Non-integrated defect relation for meromorphic maps of complex Kähler manifolds into $\mathbf{P}^{N_{1}}(\mathbf{C}) \times \cdots \times \mathbf{P}^{N_{k}}(\mathbf{C})$, Japanese J. Math., 11 (1985), 233-264.

[G1] P. Griffiths, Entire holomorphic mappings in one and several complex variables, Ann. Math. Studies 85, Princeton Univ. Press, Princeton, N. J. (1976).

[H1] W. K. Hayman, Meromorphic Functions, Oxford Univ. Press, Oxford, (1964).

[N1] R. Nevanlinna, Einige Eindeutigkeitssätze in der Theorie der meromorphen Funktionen, Acta. Math., 48 (1926), 367-391.

[N2] _ Zur Theorie der meromorphen Funktionen, Acta. Math., 46 (1925), $1-99$.

[P1] G. Pólya, Bestimmung einer ganzen Funktion endlichen Geschlechts durch viererlei Stellen, Math. Tidsskrift B. Kobenhavn, (1921), 16-21.

[S1] E. M. Schmid, Some theorems on value distribution of meromorphic functions, Math. Z., 120 (1971), 61-92.

[S2] B. V. Shabat, Distribution of values of holomorphic mappings, Transl. of Math. Mono. Vol. 61, Amer. Math. Soc., 1985.

[S3] B. Shiffman, Nevanlinna defect relations for singular divisors, Invent. Math., 31 (1975), 155-182.

[S4] Introduction to the Carlson-Griffiths equidistribution theory, Lecture Notes in Math., vol. 981, Springer-Verlag, 1983.

[S5] L. Smiley, Dependence theorems for meromorphic maps, Notre Dame, thesis, 1979. 
[S6] , Geometric conditions for unicity of holomorphic curves, Contemporary Math., vol. 25, Amer. Math. Soc., (1983), 149-154.

[S7] W. Stoll, Value distribution on parabolic spaces, Springer Lecture Notes in Math., 600 (1977).

[S8] , Introduction to value distribution theory of meromorphic maps, in Complex Analysis, Springer Lecture Notes in Math., 950 (1982), 210-359.

[S9] - The Ahlfors-Weyl theory of meromorphic maps on parabolic manifolds, Springer Lecture Notes in Math., 981 (1983), 101-219.

[S10] - Value distribution theory for meromorphic maps, Aspects of Mathematics, E7 (1985), Vieweg-Verlag.

[S11] - to appear.

[V1] A. Vitter, The lemma of the logarithmic derivative in several complex variables, Duke Math. J., 44 (1977), 89-104.

[W1] H. Weyl and J. Weyl, Meromorphic functions and analytic curves, Annals of Math. Studies 12, Princeton Univ. Press, Princeton, N. J., 1943.

Received April 13, 1987.

THE JOHNS Hopkins UNIVERSITY

BALTIMORE, MD 21218 


\section{PACIFIC JOURNAL OF MATHEMATICS \\ EDITORS}

\author{
V. S. VARADARAJAN \\ (Managing Editor) \\ University of California \\ Los Angeles, CA 90024 \\ HERBERT CLEMENS \\ University of Utah \\ Salt Lake City, UT 84112 \\ R. FINN \\ Stanford University \\ Stanford, CA 94305
}

\author{
HERMANN FLASCHKA \\ University of Arizona \\ Tucson, AZ 85721
}

vAUGHAN F. R. JONES

University of California

Berkeley, CA 94720

STEVENKERCKH OFF

Stanford University

Stanford, CA 94305

\author{
ROBION KIRBY \\ University of California \\ Berkeley, CA 94720 \\ C. C. MOORE \\ University of California \\ Berkeley, CA 94720 \\ HAROLD T ARK
}

University of California, San Diego La Jolla, CA 92093

\section{ASSOCIATE EDITORS}
R. ARENS
E. F. BECKENBACH
B. H. NEUMANN
F. WOLF
K. YOSHIDA

(1906-1982)

\section{SUPPORTING INSTITUTIONS}

\author{
UNIVERSITY OF ARIZONA \\ UNIVERSITY OF BRITISH COLUMBIA \\ CALIFORNIA INSTITUTE OF TECHNOLOGY \\ UNIVERSITY OF CALIFORNIA \\ MONTANA STATE UNIVERSITY \\ UNIVERSITY OF NEVADA, RENO \\ NEW MEXICO STATE UNIVERSITY \\ OREGON STATE UNIVERSITY
}

\author{
UNIVERSITY OF OREGON \\ UNIVERSITY OF SOUTHERN CALIFORNIA \\ STANFORD UNIVERSITY \\ UNIVERSITY OF HAWAII \\ UNIVERSITY OF TOKYO \\ UNIVERSITY OF UTAH \\ WASHINGTON STATE UNIVERSITY \\ UNIVERSITY OF WASHINGTON
}

The Supporting Institutions listed above contribute to the cost of publication of this Journal, but they are not owners or publishers and have no responsibility for its content or policies.

Mathematical papers intended for publication in the Pacific Journal of Mathematics should be in typed form or offset-reproduced (not dittoed), double spaced with large margins. Please do not use built up fractions in the text of the manuscript. However, you may use them in the displayed equations. Underline Greek letters in red, German in green, and script in blue. The first paragraph must be capable of being used separately as a synopsis of the entire paper. In particular it should contain no bibliographic references. Please propose a heading for the odd numbered pages of less than 35 characters. Manuscripts, in triplicate, may be sent to any one of the editors. Please classify according to the scheme of Math. Reviews, Index to Vol. 39. Supply name and address of author to whom proofs should be sent. All other communications should be addressed to the managing editor, or Elaine Barth, University of California, Los Angeles, California 90024.

There are page-charges associated with articles appearing in the Pacific Journal of Mathematics. These charges are expected to be paid by the author's University, Government Agency or Company. If the author or authors do not have access to such Institutional support these charges are waived. Single authors will receive 50 free reprints; joint authors will receive a total of 100 free reprints. Additional copies may be obtained at cost in multiples of 50 .

The Pacific Journal of Mathematics is issued monthly as of January 1966. Regular subscription rate: $\$ 190.00$ a year (5 Vols., 10 issues). Special rate: $\$ 95.00$ a year to individual members of supporting institutions.

Subscriptions, orders for numbers issued in the last three calendar years, and changes of address should be sent to Pacific Journal of Mathematics, P.O. Box 969, Carmel Valley, CA 93924, U.S.A. Old back numbers obtainable from Kraus Periodicals Co., Route 100, Millwood, NY 10546.

The Pacific Journal of Mathematics at P.O. Box 969, Carmel Valley, CA 93924 (ISSN 0030-8730) publishes 5 volumes per year. Application to mail at Second-class postage rates is pending at Carmel Valley, California, and additional mailing offices. Postmaster: send address changes to Pacific Journal of Mathematics, P.O. Box 969, Carmel Valley, CA 93924.

\section{PUBLISHED BY PACIFIC JOURNAL OF MATHEMATICS, A NON-PROFIT CORPORATION} Copyright (C) 1988 by Pacific Journal of Mathematics 


\section{Pacific Journal of Mathematics}

Vol. 135, No. $2 \quad$ October, 1988

Waleed A. Al-Salam and Mourad Ismail, $q$-beta integrals and the

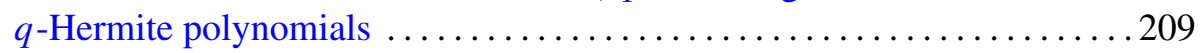

Johnny E. Brown, On the Ilieff-Sendov conjecture $\ldots \ldots \ldots \ldots \ldots \ldots \ldots 223$

Lawrence Jay Corwin and Frederick Paul Greenleaf, Spectrum and

multiplicities for restrictions of unitary representations in nilpotent Lie

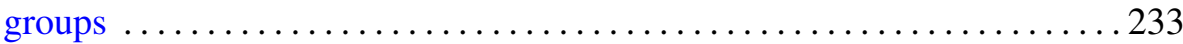

Robert Jay Daverman, 1-dimensional phenomena in cell-like mappings on 3-manifolds .......................................... 269

P. D. T. A. Elliott, A localized Erdős-Wintner theorem .............. 287

Richard John Gardner, Relative width measures and the plank problem . . . 299

F. Garibay, Peter Abraham Greenberg, L. Reséndis and Juan José

Rivaud, The geometry of sum-preserving permutations ............313

Shanyu Ji, Uniqueness problem without multiplicities in value distribution

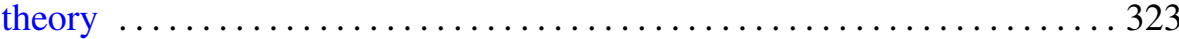

Igal Megory-Cohen, Finite-dimensional representation of classical

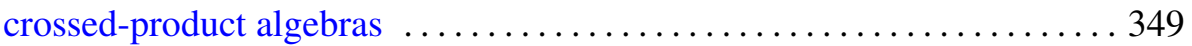

Mirko Navara, Pavel Pták and Vladimír Rogalewicz, Enlargements of

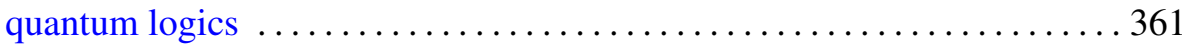

Claudio Nebbia, Amenability and Kunze-Stein property for groups acting

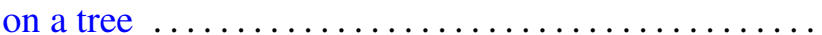

Chull Park and David Lee Skoug, A simple formula for conditional Wiener

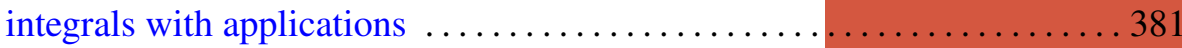

Ronald Scott Irving and Brad Shelton, Correction to: "Loewy series and simple projective modules in the category $\mathrm{O}_{s} " \ldots .$.

Robert Tijdeman and Lian Xiang Wang, Correction to: "Sums of products

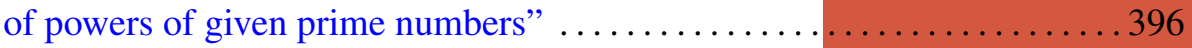

